\title{
Nonmesonic weak decay of the hypertriton
}

\author{
J. Golak, ${ }^{2}$ K. Miyagawa, ${ }^{3}$ H. Kamada,${ }^{4}$ H. Witała, ${ }^{2}$ W. Glöckle,${ }^{1}$ A. Parreño, ${ }^{5}$ A. Ramos,${ }^{5}$ and C. Bennhold ${ }^{6}$ \\ ${ }^{1}$ Institut für Theoretische Physik II, Ruhr Universität Bochum, D-44780 Bochum, Germany \\ ${ }^{2}$ Institut of Physics, Jagellonian University, PL 30059 Cracow, Poland \\ ${ }^{3}$ Department of Applied Physics, Okayama University of Science, Ridai-cho Okayama 700, Japan \\ ${ }^{4}$ Paul Scherrer Institute, CH-5232 Villigen PSI, Switzerland \\ ${ }^{5}$ Departament d'Estructura i Constituents de la Matèria, Facultat de Física, Diagonal 647, E-08028, Spain \\ ${ }^{6}$ Center for Nuclear Studies, The George Washington University, Washington, D.C., 20052
}

(Received 4 December 1996)

\begin{abstract}
The nonmesonic decay of the hypertriton is calculated based on a hypertriton wave function and $3 N$ scattering states, which are rigorous solutions of three-body Faddeev equations using realistic $N N$ and hyperon-nucleon interactions. The pion exchange together with heavier meson exchanges for the $\Lambda N \rightarrow N N$ transition is considered. The total nonmesonic decay rate is found to be $0.5 \%$ of the free $\Lambda$ decay rate. Integrated as well as differential decay rates are given. The $p$ - and $n$-induced decays are discussed thoroughly and it is shown that the corresponding total rates cannot be measured individually. [S0556-2813(97)03905-8]
\end{abstract}

PACS number(s): 21.80. $+\mathrm{a}, 21.45 .+\mathrm{v}, 23.40 .-\mathrm{s}, 27.10 .+\mathrm{h}$

\section{INTRODUCTION}

The hypertriton consisting of a neutron, proton, and a $\Lambda$ or $\Sigma$, which strongly convert into each other, is bound against $\Lambda$-deuteron decay by $0.13 \pm 0.05 \mathrm{MeV}$. Recently that number could be reproduced [1] by solving the Faddeev equations based on realistic $N N$ forces and the Nijmegen hyperon-nucleon interaction [2]. The resulting wave function has all two-body correlations exactly built in as enforced by the various two-body forces. As the lightest hypernucleus the hypertriton plays the same role in hypernuclear physics that the deuteron does in nuclear physics. However, in contrast to the deuteron, the hypertriton is subject to the weak decay and has a lifetime comparable to that of the free $\Lambda$, $\tau_{\Lambda}=2.63 \times 10^{-10} \mathrm{sec}$. The first data on light hypernuclear lifetimes have been obtained using bubble chamber experiments and emulsion works which in most cases only detected the mesonic decay modes. These measurements suffered from low precision, very poor statistics and difficulties with particle identification, leading to fairly large error bars. Along with the mesonic two-body decay mode ${ }_{\Lambda}^{3} \mathrm{H} \rightarrow \pi^{-}\left(\pi^{0}\right)+{ }^{3} \mathrm{He}\left({ }^{3} \mathrm{H}\right)$ there are the corresponding mesonic multibody decay modes ${ }_{\Lambda}^{3} \mathrm{H} \rightarrow \pi^{-}\left(\pi^{0}\right)+d+p(n)$ and ${ }_{\Lambda}^{3} \mathrm{H} \rightarrow \pi^{-}\left(\pi^{0}\right)+p+n+p(n)$. The most precise experiment to date for the combined two- and multibody decay modes gave a value of $\tau=(2.28+0.46-0.33) \times 10^{-10} \mathrm{sec}[3]$. Furthermore, a similar measurement was also able to estimate the decay branching ratio $\Gamma\left({ }_{\Lambda}^{3} \mathrm{H} \rightarrow \pi^{-}+{ }^{3} \mathrm{He}\right) / \Gamma\left({ }_{\Lambda}^{3} \mathrm{H} \rightarrow\right.$ all $\pi^{-}$mesonic modes) as $0.30 \pm 0.07$.

Besides the mesonic decay channels there are also two nonmesonic modes, ${ }_{\Lambda}^{3} \mathrm{H} \rightarrow d+p$ and ${ }_{\Lambda}^{3} \mathrm{H} \rightarrow p+p+n$. While it is well known that these channels dominate the weak decay of heavy hypernuclei they are expected to be rare for the hypertriton since the mesonic modes are not Pauli suppressed [4]. As a first step this two-baryon decay mode $\Lambda N \rightarrow N N$ can be understood in terms of the free-space decay mechanism $\Lambda \rightarrow \pi N$ with virtual pion that is absorbed on a second nucleon bound in the hypernucleus [5]. However, the large momentum transfer involved in the reaction leads to a mechanism that is sensitive to the short distance behavior of the amplitude and allows for the exchange of heavier mesons. The production of these mesons would be below threshold for the free-space $\Lambda$ decay, but they can contribute through virtual exchange in a two-baryon decay channel.

The weak nonmesonic decay channel is important since it allows access to the fundamental aspects of the four-fermion, strangeness changing weak interaction. In general, starting with the standard model electroweak Hamiltonian and taking into account QCD corrections at short distances yields an effective weak $V-A$ interaction that could presumably predict the relative strength of the $\Delta S=0$ and $\Delta S=1$ transition. Thus, hadronic weak matrix elements of the form $\left\langle M B^{\prime}\left|H_{w}\right| B\right\rangle$ can be calculated [6]. Using these weak vertices as a starting point for effective nuclear two-body operators that are then implanted into the nucleus with the usual nuclear many-body wave functions provides the testing ground for the effective interaction.

Parity violation in hadronic systems represents a unique tool to study aspects of the nonleptonic weak interaction between hadrons. The nonmesonic process resembles the weak $\Delta S=0$ nucleon-nucleon interaction that has been explored experimentally in parity-violating $N N$ scattering measurements. However, the $\Lambda N \rightarrow N N$ two-body decay mode can explore both the parity-conserving (PC) and the parityviolating (PV) sector of the $\Delta S=1$ weak baryon-baryon interaction while in the weak $N N$ system one is limited to the weak PV interaction. A number of theoretical approaches to the $\Lambda N \rightarrow N N$ decay mode have been developed over the last 30 years which are more extensively reviewed in Ref. [5]. The $\Delta S=0$ weak nucleon-nucleon interaction at low and intermediate energies has generally been described in a meson exchange model involving one strong interaction vertex and one weak one; the same approach can be used for a microscopic description of the $\Delta S=1 \Lambda N \rightarrow N N$ mechanism.

A recently completed major study of the nonmesonic decay of $p$-shell hypernuclei [7] found that proper short-range correlations in the initial and final state are of major impor- 
tance in predicting decay rates and asymmetry observables. However, in a shell-model framework bound state wave functions, spectroscopic factors, short-range correlations, and final state interactions do not all originate from the same underlying dynamics and, therefore, introduce approximations that may be difficult to quantify. Since the aim of investigating the nonmesonic decay is to extract information on hadronic weak vertices from the $\Lambda N \rightarrow N N$ process, the decay of few-body hypernuclei offers a window since all nuclear structure ingredients are derived from the same baryon-baryon interaction.

It thus appears worthwhile to repeat a former study [4] on the weak nonmesonic decay of the hypertriton, where a simplified uncorrelated deuteron- $\Lambda$ wave function has been used. We expect that correlations should play an important role, since the mesons emitted by the weak hyperon-nucleon transition are reabsorbed by the nucleons. The resulting meson-exchange operator acts like a two-body force and consequently probes the hypertriton wave function in its dependence on the pair distance between a hyperon and a nucleon. Furthermore, the final three nucleons will interact strongly with each other, which might influence significantly the decay process. This dynamical ingredient has been neglected in Ref. [4] and will now be fully incorporated.

In Sec. II the theoretical formalism for the evaluation of the decay matrix element will be given. Section III describes the necessary technicalities. A special section, Sec. IV, is devoted to the meson exchange operator. We present our results in Sec. V. We summarize and conclude in Sec. VI.

\section{FORMALISM}

There are two nonmesonic decay channels

$$
{ }_{\Lambda}^{3} H \rightarrow\left\{\begin{array}{c}
n+d \\
n+n+p
\end{array}\right.
$$

According to standard rules, the partial decay probabilities in the total momentum zero frame are

$$
\begin{aligned}
& d \Gamma^{n+d}=\frac{1}{2} \sum_{m m_{N} m_{d}}\left|\left\langle\Psi_{\vec{k}_{N} \vec{k}_{d} m_{N} m_{d}}^{(-)}|\hat{O}| \Psi_{\Lambda}^{3} \mathrm{H} m\right\rangle\right|^{2} \\
& \times d \vec{k}_{N} d \vec{k}_{d} 2 \pi \delta\left(\vec{k}_{N}+\vec{k}_{d}\right) \\
& \times \delta\left(M_{\Lambda}^{3} \mathrm{H}-M_{N}-M_{d}-\frac{\vec{k}_{N}^{2}}{2 M_{N}}-\frac{\vec{k}_{d}^{2}}{2 M_{d}}\right)
\end{aligned}
$$

and

$$
\begin{aligned}
& d \Gamma^{n+n+p}=\frac{1}{2} \sum_{m m_{1} m_{2} m_{3}}\left|\left\langle\Psi_{\vec{k}_{1} \vec{k}_{2} \vec{k}_{3} m_{1} m_{2} m_{3}}^{(-)}|\hat{O}| \Psi_{\Lambda} \mathrm{H} m\right\rangle\right|^{2} \\
& \times d \vec{k}_{1} d \vec{k}_{2} d \vec{k}_{3} 2 \pi \delta\left(\vec{k}_{1}+\vec{k}_{2}+\vec{k}_{3}\right) \\
& \times \delta\left(M_{\Lambda}^{3}{ }^{H}-3 M_{N}-\frac{\vec{k}_{1}^{2}}{2 M_{N}}-\frac{\vec{k}_{2}^{2}}{2 M_{N}}-\frac{\vec{k}_{3}^{2}}{2 M_{N}}\right),
\end{aligned}
$$

where $\Psi^{(-)}$are appropriate three-nucleon scattering states, $\hat{O}$ the transition operator, and $\Psi_{\Lambda}^{3} \mathrm{H}$ the hypertriton wave function. We use nonrelativistic notation throughout. The binding energies are defined as usual in terms of various masses as

$$
M_{\Lambda}^{3} \mathrm{H}^{2}=2 M_{N}+M_{\Lambda}+\epsilon, \quad M_{d}=2 M_{N}+\epsilon_{d} .
$$

Further, we introduce Jacobi momenta for the final $3 N$ states,

$$
\vec{p}=\frac{1}{2}\left(\vec{k}_{1}-\vec{k}_{2}\right), \vec{q}=\frac{2}{3}\left[\vec{k}_{3}-\frac{1}{2}\left(\vec{k}_{1}+\vec{k}_{2}\right)\right]
$$

and identify for the $n d$ breakup $\vec{k}_{3}=\vec{k}_{N}$ and $\vec{k}_{1}+\vec{k}_{2}=\vec{k}_{d}$. Then some simple algebra leads to

$$
d \Gamma^{n+d}=\frac{1}{2} \sum_{m m_{N} m_{d}}\left|\left\langle\Psi_{q_{0} m_{N} m_{d}}^{(-)}|\hat{O}| \Psi_{\Lambda}^{3} \mathrm{H} m\right\rangle\right|^{2} 2 \pi \frac{2 M_{N}}{3} q_{0} d \hat{q},
$$

with

$$
q_{0}=\sqrt{\frac{4 M_{N}}{3}\left(M_{\Lambda}-M_{N}+\epsilon-\epsilon_{d}\right)} .
$$

Because of the averaging over spin directions the matrix element squared is independent of $\hat{q}_{0}$ and we get just a number:

$$
\Gamma^{n+d}=8 \pi^{2} \frac{2 M_{N}}{3} q_{0} \frac{1}{2} \sum_{m m_{N} m_{d}}\left|\left\langle\Psi_{q_{0} m_{N} m_{d}}^{(-)}|\hat{O}| \Psi_{\Lambda^{3} \mathrm{H} m}\right\rangle\right|^{2} .
$$

Similar steps lead to

$$
\begin{aligned}
d \Gamma^{n+n+p}= & \frac{1}{2} \sum_{m m_{1} m_{2} m_{3}}\left|\left\langle\Psi_{\vec{p} q m_{1} m_{2} m_{3}}^{(-)}|\hat{O}| \Psi_{\Lambda}^{3} \mathrm{H} m\right\rangle\right|^{2} \\
& \times 2 \pi \frac{2 M_{N}}{3} q d \hat{q} d \hat{p} p^{2} d p
\end{aligned}
$$

with

$$
q=\sqrt{\frac{4 M_{N}}{3}\left(M_{\Lambda}-M_{N}+\epsilon-\frac{\vec{p}^{2}}{M_{N}}\right)}
$$

Again the spin-averaged part depends only on the angle $\theta$ between $\hat{p}$ and $\hat{q}$, thus

$$
\begin{aligned}
& d \Gamma^{n+n+p}=16 \pi^{3} \frac{2}{3} M_{N} q p^{2} d p \sin \theta d \theta \\
& \times \frac{1}{2} \sum_{m m_{1} m_{2} m_{3}}\left|\left\langle\Psi_{\vec{p} q m_{1} m_{2} m_{3}}^{(-)}|\hat{O}| \Psi_{{ }_{\Lambda} \mathrm{H} m}\right\rangle\right|^{2}
\end{aligned}
$$

This form is convenient for the integration to determine the total ( $n n p)$ decay rate. For the display of the angular and 


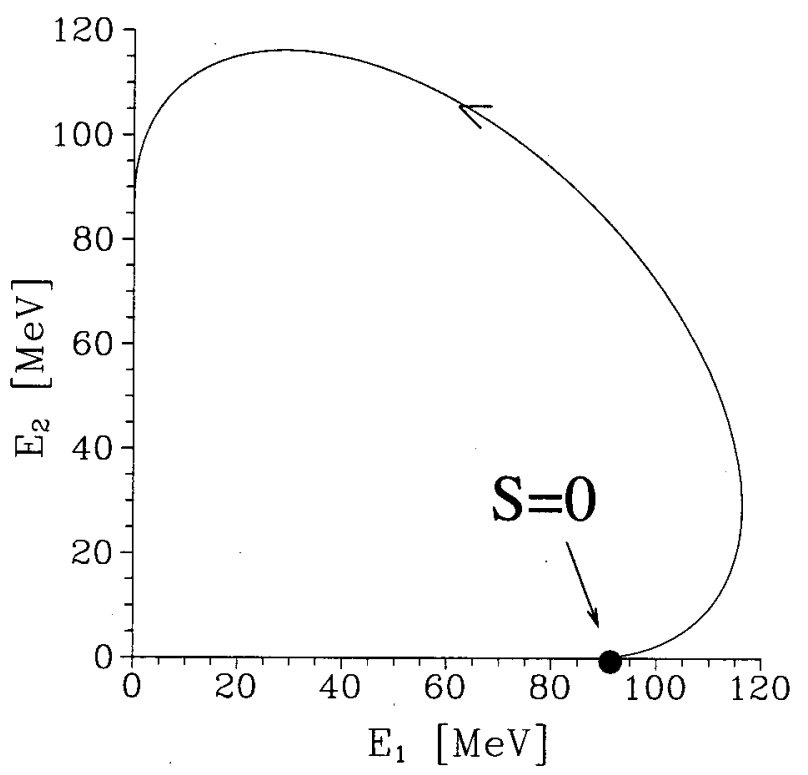

FIG. 1. Locus for kinematically allowed events in the $E_{1}-E_{2}$ plane and $\Theta_{12}=180^{\circ}$ together with our definition of the choice for the arclength $S=0$. From that point on $S$ is evaluated for each point on the locus in the counterclockwise sense.

energy distribution of the three nucleons, the following, equivalent form [6] is more convenient:

$$
\begin{aligned}
d \Gamma^{n+n+p}= & \frac{1}{2} \sum_{m m_{1} m_{2} m_{3}}\left|\left\langle\Psi_{\overrightarrow{p q} m_{1} m_{2} m_{3}}^{(-)}|\hat{O}| \Psi_{{ }_{\Lambda} \mathrm{H} m}\right\rangle\right|^{2} \\
& \times 2 \pi d \hat{k}_{1} d \hat{k}_{2} d S \\
& \times \frac{M_{N}^{2} k_{1}^{2} k_{2}^{2}}{\sqrt{k_{1}^{2}\left(2 k_{2}+\vec{k}_{1} \cdot \hat{k}_{2}\right)^{2}+k_{2}^{2}\left(2 k_{1}+\vec{k}_{2} \cdot \hat{k}_{1}\right)^{2}}} .
\end{aligned}
$$

Here $\hat{k}_{1}$ and $\hat{k}_{2}$ denote the directions of two detected nucleons. That choice of four angles relates the lab energies $E_{1}$ and $E_{2}$ kinematically through energy and momentum conservation, as shown in the example of an interparticle angle $\Theta_{12}=180^{\circ}$ in Fig. 1. A point on that curve can be defined through the arclength $S$ measured from some conveniently chosen point. Our choice for $S=0$ is shown in Fig. 1. Thus, instead of expressing the fivefold differential cross section with respect to $d E_{1}$, for instance, we have chosen $d S$ in Eq. (11).
In Eq. (11) the necessary additional information, whether the detected particles 1 and 2 are a proton-neutron pair or two neutrons, has been dropped for the sake of a simple notation. The final scattering state carries, however, additional isotopic spin quantum numbers $\nu_{1} \nu_{2} \nu_{3}$.

Throughout the paper the normalization of the momentum states is always like $\left\langle\vec{k} \mid \vec{k}^{\prime}\right\rangle=\delta^{3}\left(\vec{k}-\vec{k}^{\prime}\right)$.

The operator $\hat{O}$ is of two-body character and acts between the $\Lambda$ and a nucleon. In the hypertriton wave function let us denote the $\Lambda$ to be particle 1 , then

$$
\hat{O}=\sum_{i=2,3} \hat{O}(1, i)
$$

Because of the antisymmetry of the hypertriton state and the scattering states with respect to the two nucleons 2 and 3 the nuclear matrix element simplifies to

$$
\left\langle\Psi^{(-)}|\hat{O}| \Psi_{\Lambda^{3}}\right\rangle=2\left\langle\Psi^{(-)}|\hat{O}(1,2)| \Psi_{{ }^{3}} \mathrm{H}\right\rangle .
$$

The exact inclusion of the final state interactions among the three final nucleons can be performed in analogy to electron scattering on ${ }^{3} \mathrm{He}[10]$. We exemplify it for the $n n p$ breakup process. For our notation in general we refer to [11].

The scattering state $\Psi^{(-)} \equiv \Psi_{p q}^{(-)}$is Faddeev decomposed

$$
\Psi^{(-)}=(1+P) \psi^{(-)},
$$

where $P$ is the sum of a cyclical and anticyclical permutation of three objects and $\psi^{(-)}$is one Faddeev component. It obeys the Faddeev equation

$$
\psi^{(-)}=\phi^{(-)}+G_{0}^{(-)} t^{(-)} P \psi^{(-)}
$$

with

$$
\begin{gathered}
\phi^{(-)}=\left(1+G_{0}^{(-)} t^{(-)}\right) \phi_{0}^{a}, \\
\phi_{0}^{a}=\frac{1}{\sqrt{3 !}}\left(1-P_{12}\right)\left|\phi_{0}\right\rangle \equiv \frac{1}{\sqrt{6}}\left(1-P_{12}\right)|\vec{p}\rangle|\vec{q}\rangle .
\end{gathered}
$$

Here $G_{0}^{(-)}$is the free three-nucleon propagator, $t^{(-)}$the $N N$ (off-shell) $t$ matrix, and $1 / \sqrt{6}$ takes care of the identity of the three nucleons. Note that $P_{12}$ acts in the two-body subsystem described by the relative momentum $\vec{p}$. Let us now insert Eqs. (14), (15), and (16) into the nuclear matrix element

$$
\begin{aligned}
& \left\langle\Psi^{(-)}|\hat{O}(1,2)| \Psi_{\Lambda^{3}}\right\rangle=\left\langle\psi^{(-)}|(1+P) \hat{O}(1,2)| \Psi_{{ }^{3}} \mathrm{H}\right\rangle \\
& =\left\langle\phi^{(-)}|(1+P) \hat{O}(1,2)| \Psi_{{ }^{3}}{ }\right\rangle+\left\langle\psi^{(-)}\left|P t G_{0}(1+P) \hat{O}(1,2)\right| \Psi_{{ }^{3}} \mathrm{H}\right\rangle \\
& =\left\langle\phi_{0}^{a}\left|\left(1+t G_{0}\right)(1+P) \hat{O}(1,2)\right| \Psi_{\Lambda}{ }_{\mathrm{H}}\right\rangle+\left\langle\phi_{0}^{a}\left|\left(1+t G_{0}\right) P t G_{0}(1+P) \hat{O}(1,2)\right| \Psi_{{ }^{3} \mathrm{H}}\right\rangle \\
& +\left\langle\phi_{0}^{a}\left|\left(1+t G_{0}\right) \operatorname{Pt}_{0} P t G_{0}(1+P) \hat{O}(1,2)\right| \Psi_{\Lambda}^{3} \mathrm{H}\right\rangle+\cdots .
\end{aligned}
$$




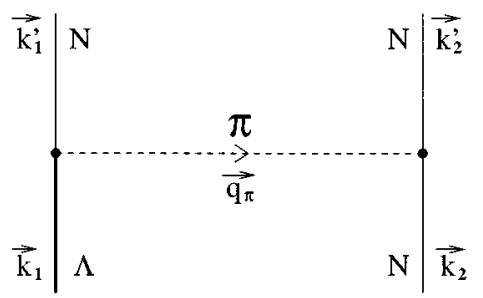

FIG. 2. The $\pi$-induced $\Lambda N \rightarrow N N$ exchange process of Eqs. (23) and (24).

In the last equality we iterated Eq. (15). It is then easily seen that this can be put into the form

$$
\begin{aligned}
& \left\langle\Psi_{\vec{p} q}^{(-)}|\hat{O}(1,2)| \Psi_{{ }_{\Lambda}} \mathrm{H}\right\rangle \\
& =\left\langle\phi_{0}^{a}|(1+P) \hat{O}(1,2)| \Psi_{{ }_{\Lambda}} \mathrm{H}\right\rangle+\left\langle\phi_{0}^{a}|(1+P)| U\right\rangle,
\end{aligned}
$$

where $|U\rangle$ obeys the Faddeev equation

$$
|U\rangle=t G_{0}(1+P) \hat{O}(1,2)\left|\Psi_{\Lambda^{3}}\right\rangle+t G_{0} P|U\rangle .
$$

Note the driving term of that integral equation contains the operator $\hat{O}(1,2)$ applied to the hypertriton bound state $\left|\Psi_{{ }_{3} \mathrm{H}}\right\rangle$ and it also includes rescattering terms of first order in $t$. The pure plane wave impulse approximation is the first term on the right-hand side of Eq. (19).

A similar reduction yields for the $n d$ breakup process

$$
\left\langle\Psi_{\vec{q}_{0}}^{(-)}|\hat{O}(1,2)| \Psi_{\Lambda^{3}}\right\rangle=\left\langle\phi|(1+P) \hat{O}(1,2)| \Psi_{{ }^{3} \mathrm{H}}\right\rangle+\langle\phi|P| U\rangle,
$$

where now $|\phi\rangle$ contains the deuteron state $\left|\varphi_{d}\right\rangle$ :

$$
|\phi\rangle=\left|\varphi_{d}\right\rangle\left|\vec{q}_{0}\right\rangle
$$

and the same state $|U\rangle$ appears.

Using the weak transition operator $\hat{O}(1,2)$ in the context of a strictly nonrelativistic framework requires some approximations, which we would like to describe in the example of pion exchange $[5,7]$. According to Fig. 2 the transition operator is

$$
\begin{aligned}
\hat{O}(1,2)= & i^{2} \bar{u}\left(\vec{k}_{2}^{\prime}\right) g_{N N \pi} \gamma_{5} u\left(\vec{k}_{2}\right) \frac{F^{2}\left(q_{\pi}^{2}\right)}{q_{\pi}^{2}-m_{\pi}^{2}} \bar{u}\left(\vec{k}_{1}^{\prime}\right) G_{F} m_{\pi}^{2}\left(A_{\pi}\right. \\
& \left.+B_{\pi} \gamma_{5}\right) u_{\Lambda}\left(\vec{k}_{1}\right) .
\end{aligned}
$$

Here $u$ and $\bar{u}$ are the usual Dirac spinors, $g_{N N \pi}$ the strong coupling constant for the $N N \pi$ vertex, and $G_{F} m_{\pi}^{2}$ $=2.21 \times 10^{-7}$ the weak coupling constant. The constants $A_{\pi}=1.05$ and $B_{\pi}=-7.15$, which determine the strength of the parity-violating and parity-conserving amplitudes, respectively, have been adjusted to reproduce the decay observables of the free $\Lambda$ particle [8]. We assume the same form factor $F$ at the two vertices (the strong and the weak one). In the nonrelativistic reduction at the weak vertex the nucleon mass, $M_{N}$, and the $\Lambda$ mass, $M_{\Lambda}$, are replaced by their average, $\bar{M}$. Then one finds

$$
\hat{O}(1,2) \rightarrow-G_{F} m_{\pi}^{2} \frac{g_{N N \pi}}{2 M_{N}} \frac{F^{2}\left(\vec{q}_{\pi}^{2}\right)}{\vec{q}_{\pi}^{2}+m_{\pi}^{2}} \vec{\sigma}_{2} \cdot \vec{q}_{\pi}\left(A_{\pi}+\frac{B_{\pi}}{2 \bar{M}} \vec{\sigma}_{\Lambda} \cdot \vec{q}_{\pi}\right)
$$

with

$$
\vec{q}_{\pi}=\vec{k}_{1}-\vec{k}_{1}^{\prime}=\vec{k}_{2}^{\prime}-\vec{k}_{2}
$$

We have to use two types of Jacobi momenta, one set referring to the hypertriton composed of $\Lambda N N$ and another set for the final state of three nucleons. The latter ones have been already defined in Eq. (4) and will be denoted by $\vec{p}^{\prime}$ and $\vec{q}^{\prime}$. The ones for the hypertriton are

$$
\vec{p}=\frac{M_{N} \vec{k}_{1}-M_{\Lambda} \vec{k}_{2}}{M_{N}+M_{\Lambda}}
$$

$$
\vec{q}=\frac{\left(M_{N}+M_{\Lambda}\right) \vec{k}_{3}-M_{N}\left(\vec{k}_{1}+\vec{k}_{2}\right)}{2 M_{N}+M_{\Lambda}} .
$$

Then for total momentum zero and using the spectator condition $\vec{q}=\vec{q}^{\prime}$ one has

$$
\vec{q}_{\pi}=\vec{p}-\vec{p}^{\prime}+\frac{M_{\Lambda}-M_{N}}{2\left(M_{\Lambda}+M_{N}\right)} \vec{q}
$$

As in the derivation of $\hat{O}(1,2)$ itself we also neglect here the difference $M_{\Lambda}-M_{N}$ with respect to $M_{\Lambda}+M_{N}$ and put

$$
\vec{q}_{\pi} \rightarrow \vec{p}-\vec{p}^{\prime}
$$

Then we get an ordinary two-body force, which does not depend on the momentum of the third particle (which it would if the mass difference would be included).

A final remark refers to the isospin part of the transition matrix element. At the weak vertex the $\Lambda$ has to change into a neutron or a proton by emission of a $\pi^{0}$ or $\pi^{-}$, respectively. This can be formally accomplished by setting artificially the $\Lambda$ state to be $\left|\frac{1}{2}-\frac{1}{2}\right\rangle$ in isospin and introducing $\vec{\tau}$ at the vertex. This is a well-known trick [5,7] and is in agreement with the empirical $\Delta I=\frac{1}{2}$ rule. As a consequence the two-body force (24) has to be multiplied by $\vec{\tau}_{1} \cdot \vec{\tau}_{2}$.

Now in the hypertriton the $\Lambda$ particle is treated as a strongly interacting particle and has therefore isospin zero. The isospin part of the hypertriton ( $\Lambda$ part only) is

$$
|\theta\rangle=\left|\left(\frac{1}{2} \frac{1}{2}\right) 0\right\rangle_{23}|00\rangle_{1}
$$

where the indices denote the particles and $\left(\frac{1}{2} \frac{1}{2}\right) 0$ the obvious isospin coupling for the two nucleons.

Now the action of $\vec{\tau}_{1} \cdot \vec{\tau}_{2}$ resulting from the weak transition requires the $\Lambda$ particle to be treated as $\left|\frac{1}{2}-\frac{1}{2}\right\rangle_{1}$ and consequently the isospin part of the hypertriton has to be reinterpreted as 


$$
\begin{aligned}
|\theta\rangle \rightarrow|\theta\rangle_{\text {weak }} \equiv & \left|\left(\frac{1}{2} \frac{1}{2}\right) 0\right\rangle_{23}\left|\frac{1}{2}-\frac{1}{2}\right\rangle_{1} \\
= & \frac{1}{\sqrt{2}}\left|\frac{1}{2}-\frac{1}{2}\right\rangle_{1}\left|\frac{1}{2} \frac{1}{2}\right\rangle_{2}\left|\frac{1}{2}-\frac{1}{2}\right\rangle_{3} \\
& -\frac{1}{\sqrt{2}}\left|\frac{1}{2}-\frac{1}{2}\right\rangle_{1}\left|\frac{1}{2}-\frac{1}{2}\right\rangle_{2}\left|\frac{1}{2} \frac{1}{2}\right\rangle_{3},
\end{aligned}
$$

which displays the partner (nucleon 2) of the $\Lambda$ to be a proton or a neutron, respectively.

In the nuclear matrix element one acts from the left by the isospin state of the final three-nucleon system and the $\vec{\tau}_{1} \cdot \vec{\tau}_{2}$ operator and gets for the isospin part alone

$$
\begin{gathered}
3\left\langle\left(t \frac{1}{2}\right) T M_{T}\left|\vec{\tau}_{1} \cdot \vec{\tau}_{2}\right| \theta\right\rangle_{\text {weak }} \\
=\delta_{M_{T},-1 / 2} \delta_{T, 1 / 2} \frac{\sqrt{3}}{2}\left(\sqrt{3} \delta_{t 0}+\delta_{t 1}\right) .
\end{gathered}
$$

The index 3 on the bra indicates that the isospin $t$ refers to the (12) subsystem.

We see that only total isospin $T=\frac{1}{2}$ occurs for the three final nucleons. For isoscalar meson exchanges $\vec{\tau}_{1} \cdot \vec{\tau}_{2}$ is replaced by the unit operator and the corresponding matrix element is

$$
3\left\langle\left(t \frac{1}{2}\right) T M_{T} \mid \theta\right\rangle_{\text {weak }}=\delta_{M_{T},-1 / 2} \delta_{T, 1 / 2}\left(-\delta_{t 0}+\sqrt{3} \delta_{t 1}\right) .
$$

One can artificially separate the contributions from the proton- and neutron-induced decays. This corresponds to the first and second parts on the right-hand side of Eq. (30), respectively. Keeping only one or the other, both isospins $T=\frac{1}{2}$ and $T=\frac{3}{2}$ contribute, therefore Eqs. (31) and (32) will be adequately modified. That separation into $p$-induced and $n$-induced decays will be considered in Sec. V.

\section{TECHNICALITIES}

The hypertriton state contains the $\Lambda N N$ and the $\Sigma N N$ parts. The $\Lambda-\Sigma$ conversion is crucial for the binding of the hypertriton, nevertheless the $\Sigma N N$ admixture is extremely small [1]. Thus we neglect the contribution of the $\Sigma$ decay and keep only the $\Lambda N N$ part.

In [1] the hypertriton state has been determined in a partial wave representation and we refer to [1] for the details of our notation. Here we need only the form

$$
\left|\Psi_{\Lambda^{3} \mathrm{H}}\right\rangle=\sum_{\alpha} \int d p p^{2} \int d q q^{2}|p q \alpha\rangle \Psi_{\alpha}(p q),
$$

where $p, q$ are the magnitudes of the Jacobi momenta (26) and $\alpha$ denotes the following set of discrete quantum numbers:

$$
\alpha \equiv(l s) j\left(\lambda \frac{1}{2}\right) I(j I) J\left(t \frac{1}{2}\right) T .
$$

Here $(l s) j$ describe the coupling of orbital angular momentum $l$ and total spin $s$ to the total two-body angular momentum $j$ of the $\Lambda N$ subsystem, $\left(\lambda \frac{1}{2}\right) I$ the corresponding coupling of orbital and spin angular momentum of the other nucleon to its total angular momentum $I,(j I) J$ the resulting $j I$ coupling to the total angular momentum $J$, and finally the isospin coupling of $t=\frac{1}{2}$ and $\frac{1}{2}$ to total isospin $T=0$, as described above.

Also for the evaluation of the matrix elements (19) and (21) and the solution of the Faddeev equation (20), we work in a partial wave representation, using a complete set of basis states now for three nucleons. They are again denoted as $|p q \alpha\rangle_{N}$ but adding a subscript $N$ to indicate that the Jacobi momenta are now from Eq. (4). Furthermore, one has to note that this is a subset of states antisymmetrized in the subsystem of particles 1 and 2, thus $(l+s+t)$ has to be odd.

Now projecting the Faddeev equation into the basis $|p q \alpha\rangle_{N}$ and inserting appropriate decompositions of the unity one gets

$$
\begin{aligned}
{ }_{N}\langle p q \alpha \mid U\rangle= & £ \oint_{N}\left\langle p q \alpha\left|t G_{0}(1+P)\right| p^{\prime} q^{\prime} \alpha^{\prime}\right\rangle_{N N} \\
& \times\left\langle p^{\prime} q^{\prime} \alpha^{\prime}|\hat{O}(1,2)| p^{\prime \prime} q^{\prime \prime} \alpha^{\prime \prime}\right\rangle \Psi_{\alpha^{\prime \prime}}\left(p^{\prime \prime} q^{\prime \prime}\right) \\
& +\oint_{N}\left\langle p q \alpha\left|t G_{0} P\right| p^{\prime} q^{\prime} \alpha^{\prime}\right\rangle_{N N}\left\langle p^{\prime} q^{\prime} \alpha^{\prime} \mid U\right\rangle
\end{aligned}
$$

This is a coupled set of integral equations, with a kernel part, which is well known [9] from $3 N$ scattering, and an inhomogeneous term, whose part left of $\hat{O}(1,2)$ is also familiar from electron scattering [10]. What is left as a new structure is the application of the $\hat{O}(1,2)$ matrix onto the wave function component of the hypertriton.

Now that $\hat{O}(1,2)$ matrix is obviously diagonal in the quantum numbers of the spectator nucleon:

$$
\begin{aligned}
& { }_{N}\left\langle p q \alpha|\hat{O}(1,2)| p^{\prime} q^{\prime} \alpha^{\prime}\right\rangle \\
& =\frac{\delta\left(q-q^{\prime}\right)}{q q^{\prime}} \delta_{\lambda \lambda^{\prime}} \delta_{I I^{\prime}}\left\langle p(l s) j|\hat{O}(1,2)| p^{\prime}\left(l^{\prime} s^{\prime}\right) j\right\rangle
\end{aligned}
$$

and one is left with a simple application of the two-body force onto the hypertriton in momentum space. The righthand side of Eq. (34) should contain the appropriate isospin matrix element in the three-particle space, see Eqs. (31) and (32), as a factor.

Once the amplitudes ${ }_{N}\langle p q \alpha \mid U\rangle$ are determined, the matrix elements in Eqs. (19) and (21) can be evaluated by quadratures in the manner described in [9] and references therein.

\section{THE TRANSITION OPERATOR}

On top of the $\pi$-induced transition potential described in Sec. II we include exchange potentials driven by $\eta, K, \rho$, $\omega$, and $K^{*}$ mesons. The explicit expressions for the weak and strong Hamiltonians can be found in Ref. [7].

The resulting one-boson-exchange expression in a nonrel- 
ativistic reduction for the pion is given in Eq. (24) which we rewrite here as

$$
V_{\pi}\left(\vec{q}_{\mu}\right)=-G_{F} m_{\pi}^{2} \frac{g}{2 M_{N}}\left(\hat{A}+\frac{\hat{B}}{2 \bar{M}} \vec{\sigma}_{1} \cdot \vec{q}_{\mu}\right) \frac{\vec{\sigma}_{2} \cdot \vec{q}_{\mu}}{\vec{q}_{\mu}^{2}+\mu^{2}},
$$

where $g=g_{N N \pi}$ is the strong coupling constant for the $N N \pi$ vertex, $\mu$ is the pion mass, $\vec{q}_{\mu}$ stands now for the momentum carried by the exchanged meson and the operators $\hat{A}$ and $\hat{B}$ contain the isospin dependence of the potential

$$
\hat{A}=A_{\pi} \vec{\tau}_{1} \cdot \vec{\tau}_{2}, \quad \hat{B}=B_{\pi} \vec{\tau}_{1} \cdot \vec{\tau}_{2}
$$

For pseudoscalar mesons different from the pion we have an expression analogous to Eq. (35) but making the following replacements:

$$
\begin{gathered}
g \rightarrow g_{N N \eta}, \\
\mu \rightarrow m_{\eta}, \\
\hat{A} \rightarrow A_{\eta}, \\
\hat{B} \rightarrow B_{\eta}
\end{gathered}
$$

when considering the exchange of the isoscalar $\eta$-meson, and

$$
\begin{gathered}
g \rightarrow g_{\Lambda N K}, \\
\mu \rightarrow m_{K}, \\
\hat{A} \rightarrow\left(\frac{C_{K}^{\mathrm{PV}}}{2}+D_{K}^{\mathrm{PV}}+\frac{C_{K}^{\mathrm{PV}}}{2} \vec{\tau}_{1} \cdot \vec{\tau}_{2}\right) \frac{M_{N}}{\bar{M}}, \\
\hat{B} \rightarrow\left(\frac{C_{K}^{\mathrm{PC}}}{2}+D_{K}^{\mathrm{PC}}+\frac{C_{K}^{\mathrm{PC}}}{2} \vec{\tau}_{1} \cdot \vec{\tau}_{2}\right)
\end{gathered}
$$

for the isodoublet kaon.

The factor $M_{N} / \bar{M}$ corrects for the fact that the nonrelativistic reduction of the strong $\Lambda N K$ vertex is now proportional to $\left(\vec{\sigma}_{2} \cdot \vec{q}_{\mu}\right) / 2 \bar{M}$, giving a factor $1 / \bar{M}$ instead of $1 / M_{N}$.

In the case of vector mesons as the $\rho$, one obtains [7]

$$
\begin{aligned}
V_{\rho}\left(\vec{q}_{\mu}\right)= & G_{F} m_{\pi}^{2}\left(F_{1} \hat{\alpha}-\frac{(\hat{\alpha}+\hat{\beta})\left(F_{1}+F_{2}\right)}{4 M_{N} \bar{M}}\left(\vec{\sigma}_{1} \times \vec{q}_{\mu}\right)\right. \\
& \left.\times\left(\vec{\sigma}_{2} \times \vec{q}_{\mu}\right)+i \frac{\hat{\varepsilon}\left(F_{1}+F_{2}\right)}{2 M_{N}}\left(\vec{\sigma}_{1} \times \vec{\sigma}_{2}\right) \cdot \vec{q}_{\mu}\right) \\
& \times \frac{1}{\vec{q}_{\mu}^{2}+\mu^{2}}
\end{aligned}
$$

with $\mu=m_{\rho}, F_{1}=g_{N N \rho}^{V}, F_{2}=g_{N N \rho}^{T}$ and where the operators $\hat{\alpha}, \hat{\beta}$, and $\hat{\varepsilon}$ have the following structure:

$$
\begin{aligned}
& \hat{\alpha}=\alpha_{\rho} \vec{\tau}_{1} \cdot \vec{\tau}_{2}, \\
& \hat{\beta}=\beta_{\rho} \vec{\tau}_{1} \cdot \vec{\tau}_{2}, \\
& \hat{\varepsilon}=\varepsilon_{\rho} \vec{\tau}_{1} \cdot \vec{\tau}_{2}
\end{aligned}
$$

We can get the nonrelativistic potential corresponding to the exchange of the rest of vector mesons by making the following replacements in Eq. (39):

$$
\begin{gathered}
\mu \rightarrow m_{\omega}, \\
F_{1} \rightarrow g_{N N \omega}^{V}, \\
F_{2} \rightarrow g_{N N \omega}^{T}, \\
\hat{\alpha} \rightarrow \alpha_{\omega}, \hat{\beta} \rightarrow \beta_{\omega}, \\
\hat{\varepsilon} \rightarrow \varepsilon_{\omega}
\end{gathered}
$$

for the exchange of the isoscalar $\omega$, and

$$
\begin{gathered}
\mu \rightarrow m_{K^{*}}, \\
F_{1} \rightarrow g_{\Lambda N K^{*}}^{V}, \\
F_{2} \rightarrow g_{\Lambda N K^{*}}^{T},
\end{gathered}
$$$$
\hat{\alpha} \rightarrow \frac{C_{K^{*}}^{\mathrm{PC}, V}}{2}+D_{K^{*}}^{\mathrm{PC}, V}+\frac{C_{K^{*}}^{\mathrm{PC},}}{2} \vec{\tau}_{1} \cdot \vec{\tau}_{2},
$$$$
\hat{\beta} \rightarrow \frac{C_{K^{*}}^{\mathrm{PC}, T}}{2}+D_{K^{*}}^{\mathrm{PC}, T}+\frac{C_{K^{*}}^{\mathrm{PC}, T}}{2} \vec{\tau}_{1} \cdot \vec{\tau}_{2},
$$$$
\hat{\varepsilon} \rightarrow\left(\frac{C_{K^{*}}^{\mathrm{PV}}}{2}+D_{K^{*}}^{\mathrm{PV}}+\frac{C_{K^{*}}^{\mathrm{PV}}}{2} \vec{\tau}_{1} \cdot \vec{\tau}_{2}\right) \frac{M_{N}}{\bar{M}}
$$

for the isodoublet $K^{*}$ meson.

In configuration space the potential including the exchange of all the mesons can be cast into the form

$$
\begin{aligned}
V(\vec{r})= & \sum_{i} \sum_{\alpha} V_{\alpha}^{(i)}(\vec{r})=\sum_{i} \sum_{\alpha} V_{\alpha}^{(i)}(r) \hat{O}_{\alpha} \hat{I}_{\alpha}^{(i)} \\
= & \sum_{i}\left[V_{C}^{(i)}(r) \hat{I}_{C}^{(i)}+V_{S S}^{(i)}(r) \vec{\sigma}_{1} \cdot \vec{\sigma}_{2} \hat{I}_{S S}^{(i)}\right. \\
& +V_{T}^{(i)}(r) S_{12}(\hat{r}) \hat{I}_{T}^{(i)}+\left\{n^{i} \vec{\sigma}_{2} \cdot \hat{r}+\left(1-n^{i}\right)\left[\vec{\sigma}_{1} \times \vec{\sigma}_{2}\right] \cdot \hat{r}\right\} \\
& \left.\times V_{\mathrm{PV}}^{(i)}(r) \hat{I}_{\mathrm{PV}}^{(i)}\right],
\end{aligned}
$$

where the index $i$ runs over the different mesons exchanged ( $i=1, \ldots, 6$ meaning $\pi, \rho, K, K^{*}, \eta, \omega$ ) and $\alpha$ over the different spin operators denoted by $C$ (central spin independent), $S S$ (central spin dependent), $T$ (tensor), and PV (parity violating). In the above expression, particle 1 refers to the $\Lambda$ and $n^{i}=1$ (0) for pseudoscalar (vector) mesons. For isovector mesons $(\pi, \rho)$ the isospin factor $\hat{I}_{\alpha}^{(i)}$ is $\vec{\tau}_{1} \cdot \vec{\tau}_{2}$ for all values of $\alpha$, for isoscalar mesons $(\eta, \omega)$ this factor is just 
TABLE I. Constants appearing in the weak transition potential for different mesons (in units of $G_{F} m_{\pi}^{2}$ ). The strong and weak coupling constants have been taken from Ref. [7].

\begin{tabular}{|c|c|c|c|c|}
\hline$\mu$ & $K_{C}^{\mu}$ & $K_{S S}^{\mu}$ & $K_{T}^{\mu}$ & $K_{\mathrm{PV}}^{\mu}$ \\
\hline$\pi$ & 0 & $\frac{B_{\pi}}{2 \bar{M}} \frac{g_{N N \pi}}{2 M_{N}}$ & $\frac{B_{\pi}}{2 \bar{M}} \frac{g_{N N \pi}}{2 M_{N}}$ & $A_{\pi} \frac{g_{N N \pi}}{2 M_{N}}$ \\
\hline$\eta$ & 0 & $\frac{B_{\eta}}{2 \bar{M}} \frac{g_{N N \eta}}{2 M_{N}}$ & $\frac{B_{\eta}}{2 \bar{M}} \frac{g_{N N \eta}}{2 M_{N}}$ & $A_{\eta} \frac{g_{N N \eta}}{2 M_{N}}$ \\
\hline$K$ & 0 & $\frac{1}{2 M_{N}} \frac{g_{\Lambda N K}}{2 \bar{M}}$ & $\frac{1}{2 M_{N}} \frac{g_{\Lambda N K}}{2 \bar{M}}$ & $\frac{g_{\Lambda N K}}{2 M_{N}}$ \\
\hline \multirow[t]{2}{*}{$\rho$} & $g_{N N \rho}^{V} \alpha_{\rho}$ & $\alpha_{\rho}+\beta_{\rho} g_{N N \rho}^{V}+g_{N N \rho}^{T}$ & $\alpha_{\rho}+\beta_{\rho} g_{N N \rho}^{V}+g_{N N \rho}^{T}$ & $-\varepsilon \underline{g_{N N \rho}^{V}+g_{N N \rho}^{T}}$ \\
\hline & & $2 \frac{r \bar{M}}{2 M_{N}}$ & $2 \bar{M}-2 M_{N}$ & $-\varepsilon_{\rho} \overline{2 M_{N}}$ \\
\hline \multirow[t]{2}{*}{$\omega$} & $g_{N N \omega}^{V} \alpha_{\omega}$ & $\alpha_{\omega}+\beta_{\omega} g_{N N \omega}^{V}+g_{N N \omega}^{T}$ & $\alpha_{\omega}+\beta_{\omega} g_{N N \omega}^{V}+g_{N N \omega}^{T}$ & $-\varepsilon \underline{g_{N N \omega}^{V}+g_{N N \omega}^{T}}$ \\
\hline & & $\overline { 2 \overline { M } } \longdiv { 2 M _ { N } }$ & $\overline { 2 \overline { M } } \longdiv { 2 M _ { N } }$ & $\varepsilon_{\omega} \quad 2 M_{N}$ \\
\hline$K^{*}$ & $g_{\Lambda N K^{*}}^{V}$ & $2 \frac{1}{2 M_{N}} \frac{g_{\Lambda N K *}^{V}+g_{\Lambda N K *}^{T}}{2 \bar{M}}$ & $-\frac{1}{2 M_{N}} \frac{g_{\Lambda N K *}^{V}+g_{\Lambda N K *}^{T}}{2 \bar{M}}$ & $-\frac{g_{\Lambda N K *}^{V}+g_{\Lambda N K *}^{T}}{2 M_{N}}$ \\
\hline
\end{tabular}

$\hat{1}$, and for isodoublet mesons $\left(K, K^{*}\right)$ there are contributions proportional to $\hat{1}$ and to $\vec{\tau}_{1} \cdot \vec{\tau}_{2}$ with coefficients that depend on the coupling constants and, therefore, on the spin structure piece of the potential denoted by $\alpha$.

For $K$ exchange the isospin factors in Eq. (43) are

$$
\begin{gathered}
\hat{I}_{C}^{(3)}=0, \\
\hat{I}_{S S}^{(3)}=\hat{I}_{T}^{(3)}=\frac{C_{K}^{\mathrm{PC}}}{2}+D_{K}^{\mathrm{PC}}+\frac{C_{K}^{\mathrm{PC}}}{2} \vec{\tau}_{1} \cdot \vec{\tau}_{2}, \\
\hat{I}_{\mathrm{PV}}^{(3)}=\frac{C_{K}^{\mathrm{PV}}}{2}+D_{K}^{\mathrm{PV}}+\frac{C_{K}^{\mathrm{PV}}}{2} \vec{\tau}_{1} \cdot \vec{\tau}_{2},
\end{gathered}
$$

and for $K^{*}$ exchange they are

$$
\begin{gathered}
\hat{I}_{C}^{(6)}=\frac{C_{K^{*}}^{\mathrm{PC}, V}}{2}+D_{K^{*}}^{\mathrm{PC}, V}+\frac{C_{K^{*}}^{\mathrm{PC}, V}}{2} \vec{\tau}_{1} \cdot \vec{\tau}_{2}, \\
\hat{I}_{S S}^{(6)}=\hat{I}_{T}^{(6)}=\frac{C_{K^{*}}^{\mathrm{PC}, V}+C_{K^{*}}^{\mathrm{PC}, T}}{2}+\left(D_{K^{*}}^{\mathrm{PC}, V}+D_{K^{*}}^{\mathrm{PC}, T}\right) \\
+\frac{\left(C_{K^{*}}^{\mathrm{PC}, V}+C_{K^{*}}^{\mathrm{PC}, T}\right)}{2} \vec{\tau}_{1} \cdot \vec{\tau}_{2}, \\
\hat{I}_{P V}^{(6)}=\frac{C_{K^{*}}^{\mathrm{PV}}}{2}+D_{K^{*}}^{\mathrm{PV}}+\frac{C_{K^{*}}^{\mathrm{PV}}}{2} \vec{\tau}_{1} \cdot \vec{\tau}_{2} .
\end{gathered}
$$

The different pieces $V_{\alpha}^{(i)}$, with $\alpha=C, S S, T, \mathrm{PV}$, given in Ref. [7], are reproduced here for completeness:

\begin{tabular}{|c|c|c|c|c|}
\hline \multirow[b]{2}{*}{ Meson } & \multirow[b]{2}{*}{ Strong c.c. } & \multicolumn{2}{|c|}{ Weak c.c. } & \multirow{2}{*}{$\begin{array}{c}\Lambda_{i} \\
(\mathrm{GeV})\end{array}$} \\
\hline & & $\mathrm{PC}$ & PV & \\
\hline$\pi$ & $\begin{array}{l}g_{N N \pi}=13.3 \\
g_{\Lambda \Sigma \pi}=12.0\end{array}$ & $B_{\pi}=-7.15$ & $A_{\pi}=1.05$ & 1.30 \\
\hline$\eta$ & $\begin{array}{l}g_{N N \eta}=6.40 \\
g_{\Lambda \Lambda \eta}=-6.56\end{array}$ & $B_{\eta}=-14.3$ & $A_{\eta}=1.80$ & 1.30 \\
\hline K & $\begin{array}{l}g_{\Lambda N K}=-14.1 \\
g_{N \Sigma K}=4.28\end{array}$ & $\begin{array}{l}C_{K}^{\mathrm{PC}}=-18.9 \\
D_{K}^{\mathrm{PC}}=6.63\end{array}$ & $\begin{array}{l}C_{K}^{\mathrm{PV}}=0.76 \\
D_{K}^{\mathrm{PV}}=2.09\end{array}$ & 1.20 \\
\hline$\rho$ & $\begin{array}{l}g_{N N \rho}^{V}=3.16 \\
g_{N N \rho}^{T}=13.3 \\
g_{\Lambda \Sigma \rho}^{V}=0 \\
g_{\Lambda \Sigma \rho}^{T}=11.2\end{array}$ & $\begin{array}{l}\alpha_{\rho}=-3.50 \\
\beta_{\rho}=-6.11\end{array}$ & $\mathrm{E}_{\rho}=1.09$ & 1.40 \\
\hline$\psi$ & $\begin{array}{l}g_{N N \omega}^{V}=10.5 \\
g_{N N \omega}^{T}=3.22 \\
g_{\Lambda \Lambda \omega}^{V}=7.11 \\
g_{\Lambda \Lambda \omega}^{T}=-4.04\end{array}$ & $\begin{array}{l}\alpha_{\omega}=-3.69 \\
\beta_{\omega}=-8.04\end{array}$ & $\epsilon_{\omega}=-1.33$ & 1.50 \\
\hline $\mathrm{K}^{*}$ & $\begin{aligned} g_{\Lambda N K^{*}}^{V} & =-5.47 \\
g_{\Lambda N K^{*}}^{T} & =-11.9 \\
g_{N \Sigma K^{*}} & =-3.16 \\
g_{N \Sigma K^{*}}^{T} & =6.00\end{aligned}$ & $\begin{array}{l}C_{K^{*}, V}^{\mathrm{PC}}=-3.61 \\
C_{K^{*}, T}^{\mathrm{PC}, T}=-17.9 \\
D_{K^{*}, V}^{\mathrm{PC}}=-4.89 \\
D_{K^{*}}^{\mathrm{PC}, T}=9.30\end{array}$ & $\begin{array}{l}C_{K^{*}}^{\mathrm{PV}}=-4.48 \\
D_{K^{*}}^{\mathrm{PV}}=0.60\end{array}$ & 2.20 \\
\hline
\end{tabular}

$$
\begin{gathered}
V_{C}^{(i)}(r)=K_{C}^{(i)} \frac{e^{-\mu_{i} r}}{4 \pi r} \equiv K_{C}^{(i)} V_{C}\left(r, \mu_{i}\right), \\
V_{S S}^{(i)}(r)=K_{S S}^{(i)} \frac{1}{3}\left[\mu_{i}{ }^{2} \frac{e^{-\mu_{i} r}}{4 \pi r}-\delta(r)\right] \equiv K_{S S}^{(i)} V_{S S}\left(r, \mu_{i}\right),
\end{gathered}
$$

TABLE II. Strong (Nijmegen) and weak coupling constants and cutoff parameters for the different mesons. The weak couplings are in units of $G_{F} m_{\pi}^{2}=2.21 \times 10^{-7}$. 


$$
\begin{aligned}
V_{T}^{(i)}(r) & =K_{T}^{(i)} \frac{1}{3} \mu_{i}^{2} \frac{e^{-\mu_{i} r}}{4 \pi r}\left(1+\frac{3}{\mu_{i} r}+\frac{3}{\left(\mu_{i} r\right)^{2}}\right) \\
& \equiv K_{T}^{(i)} V_{T}\left(r, \mu_{i}\right), \\
V_{\mathrm{PV}}^{(i)}(r)= & K_{\mathrm{PV}}^{(i)} \mu_{i} \frac{e^{-\mu_{i} r}}{4 \pi r}\left(1+\frac{1}{\mu_{i} r}\right) \equiv K_{\mathrm{PV}}^{(i)} V_{\mathrm{PV}}\left(r, \mu_{i}\right),
\end{aligned}
$$

where $\mu_{i}$ denotes the mass of the different mesons. The expressions for $K_{\alpha}^{(i)}$, which contain factors and coupling constants, are given in Table I. The explicit values of the strong and weak coupling constants, taken from Ref. [7], are shown in Table II.

Including monopole form factors $F_{i}\left(\vec{q}^{2}\right)=\left(\Lambda_{i}^{2}-\mu_{i}^{2}\right) /$ $\left(\Lambda_{i}^{2}+\vec{q}^{2}\right)$ at both vertices, where the value of the cutoff, $\Lambda_{i}$, depends on the meson (see Table II), leads to the following regularization of the potential:

$$
\begin{aligned}
V_{C}\left(r ; \mu_{i}\right) \rightarrow & V_{C}\left(r ; \mu_{i}\right)-V_{C}\left(r ; \Lambda_{i}\right) \\
& -\Lambda_{i} \frac{\Lambda_{i}^{2}-\mu_{i}^{2}}{2} \frac{e^{-\Lambda_{i} r}}{4 \pi}\left(1-\frac{2}{\Lambda_{i} r}\right),
\end{aligned}
$$

$$
\begin{aligned}
& V_{S S}\left(r ; \mu_{i}\right) \rightarrow V_{S S}\left(r ; \mu_{i}\right)-V_{S S}\left(r ; \Lambda_{i}\right) \\
&-\Lambda_{i} \frac{\Lambda_{i}^{2}-\mu_{i}^{2}}{2} \frac{e^{-\Lambda_{i} r}}{4 \pi}\left(1-\frac{2}{\Lambda_{i} r}\right), \\
& V_{T}\left(r ; \mu_{i}\right) \rightarrow V_{T}\left(r ; \mu_{i}\right)-V_{T}\left(r ; \Lambda_{i}\right) \\
&-\Lambda_{i} \frac{\Lambda_{i}^{2}-\mu_{i}^{2}}{2} \frac{e^{-\Lambda_{i} r}}{4 \pi}\left(1+\frac{1}{\Lambda_{i} r}\right) \\
& V_{\mathrm{PV}}\left(r ; \mu_{i}\right) \rightarrow V_{\mathrm{PV}}\left(r ; \mu_{i}\right)-V_{\mathrm{PV}}\left(r ; \Lambda_{i}\right)-\frac{\Lambda_{i}^{2}-\mu_{i}^{2}}{2} \frac{e^{-\Lambda_{i} r}}{4 \pi}
\end{aligned}
$$

where $V_{\alpha}\left(r ; \Lambda_{i}\right)$ has the same structure as $V_{\alpha}\left(r ; \mu_{i}\right)$, defined in Eqs. (46) $-(49)$, but replacing the meson mass $\mu_{i}$ by the corresponding cutoff mass $\Lambda_{i}$.

The last step is the transition into the momentum space partial wave representation (34). Of course we could have derived that directly from Eqs. (35)-(39) using the standard helicity formalism [12].

This leads to

$$
\begin{aligned}
\left\langle p(l s) j\left|V^{(i)}\right| p^{\prime}\left(l^{\prime} s^{\prime}\right) j\right\rangle= & \frac{2}{\pi} i^{\left(l^{\prime}-l\right)} \int_{0}^{\infty} d r r^{2} j_{l}(p r) V_{C}^{(i)}(r) j_{l^{\prime}}\left(p^{\prime} r\right) \delta_{l l^{\prime}} \delta_{s s^{\prime}}+\frac{2}{\pi} i^{\left(l^{\prime}-l\right)} \int_{0}^{\infty} d r r^{2} j_{l}(p r) V_{S S}^{(i)}(r) j_{l^{\prime}}\left(p^{\prime} r\right) \\
& \times\left\langle(l s) j\left|\vec{\sigma}_{1} \cdot \vec{\sigma}_{2}\right|\left(l^{\prime} s^{\prime}\right) j\right\rangle+\frac{2}{\pi} i^{\left(l^{\prime}-l\right)} \int_{0}^{\infty} d r r^{2} j_{l}(p r) V_{T}^{(i)}(r) j_{l^{\prime}}\left(p^{\prime} r\right)\left\langle(l s) j\left|S_{12}(\hat{r})\right|\left(l^{\prime} s^{\prime}\right) j\right\rangle \\
& +\frac{2}{\pi} i^{\left(l^{\prime}-l\right)} n^{i} \int_{0}^{\infty} d r r^{2} j_{l}(p r) V_{\mathrm{PV}}^{(i)}(r) j_{l^{\prime}}\left(p^{\prime} r\right)\left\langle(l s) j\left|\overrightarrow{\sigma_{2}} \cdot \hat{r}\right|\left(l^{\prime} s^{\prime}\right) j\right\rangle+\frac{2}{\pi} i^{\left(l^{\prime}-l\right)}\left(1-n^{i}\right) \\
& \times \int_{0}^{\infty} d r r^{2} j_{l}(p r) V_{\mathrm{PV}}^{(i)}(r) j_{l^{\prime}}\left(p^{\prime} r\right)\left\langle(l s) j\left|\left[\vec{\sigma}_{1} \times \vec{\sigma}_{2}\right] \cdot \hat{r}\right|\left(l^{\prime} s^{\prime}\right) j\right\rangle .
\end{aligned}
$$

The radial integrations were carried out numerically. The angular momentum parts are standard and are given, for instance, in [7].

Since our results show a strong dependence on the different meson contributions with varying signs, we would like to display the radial shapes of the four types of potentials ( $C, S S, T, \mathrm{PV})$ split into the different meson contributions. This is shown in Figs. 3(a) and 3(b) for the central spinindependent, in Figs. 4(a) and 4(b) for the central spindependent, in Figs. 5(a) and 5(b) for the tensor, and in Figs. 6(a) and 6(b) for the parity-violating channels. Note that we have represented $r^{2} V_{\alpha}^{(i)}$, where $V_{\alpha}^{(i)}$ is the potential regularized by the effect of form factors, and that the expectation value of the isospin factor for each meson and channel has also been included. As expected, we observe that the $\pi$-meson contribution is by far the one of longest range. More interesting is to note that, compared to the pion, all the other mesons play a relevant role in a wide range which extends up to about $1.5 \mathrm{fm}$. On the right-hand side of the figures we have plotted the full potential obtained when all meson contributions are added. We observe that the full potential is clearly different from the $\pi$-only one, shown on the left-hand side of the figures. We also see that in the spinindependent central channel [Figs. 3(a) and 3(b)] the contribution of the vector mesons compensate each other giving rise to a practically negligible transition potential for both isospin channels.

In Figs. 3(a) and 3(b) only the vector mesons appear since they are the ones that contribute to the spin-independent channel. These figures show that the three potentials have about the same range and their contribution is similarly relevant.

As seen in Figs. 4(a) and 4(b), the $K^{*}$ meson gives a very important contribution to the central spin-dependent channel. We also observe that, except in the intermediate range where the potentials change sign, there is a constructive interference between the pseudoscalar and vector components of each isospinlike pair $\left[(\pi, \rho),\left(K, K^{*}\right),(\eta, \omega)\right]$. Note that, in the $T=1$ channel, the $\omega$ meson lies very close to the $\rho$-meson potential. This is due to the similar value of the $\rho$ 


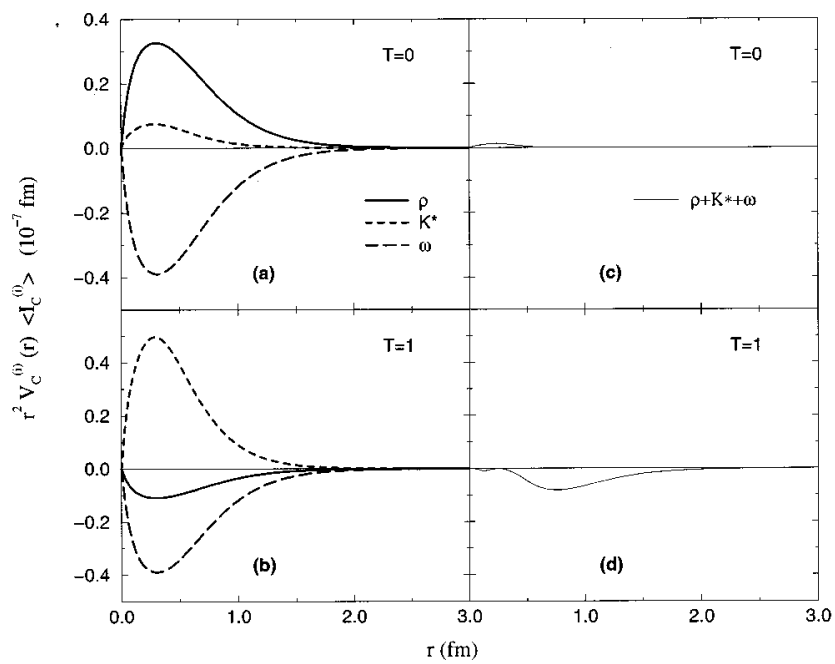

FIG. 3. Individual meson contributions to the isoscalar (a) and isovector (b) central spin independent regularized potential $r^{2} V_{C}^{\mu}(r)$. The potential obtained by adding all meson contributions for the isoscalar (c) and isovector (d) cases.

and $\omega$ masses and to the fact that the combination of strong and weak coupling constants building up $K_{S S}^{(i)}$ (see Tables I and II) gives, by chance, a very similar value. This behavior is not observed for the $T=0$ channel because, due to its isovector character, the $\rho$-meson contains an additional factor of -3 compared to the $\omega$ meson, as can be clearly seen in Fig. 4(a).

The tensor transition potential is shown in Figs. 5(a) and 5(b). In this case, we observe a destructive interference pattern for each pair of isospin-like mesons. In the $T=1$ channel the $K^{*}$ meson clearly stands out with respect to the other mesons and, for the same reasons explained above, the $\rho$ and $\omega$ contributions are again very similar.

Figures 6(a) and 6(b) show the parity-violating contributions. Here, the interference is constructive for the $(\eta, \omega)$

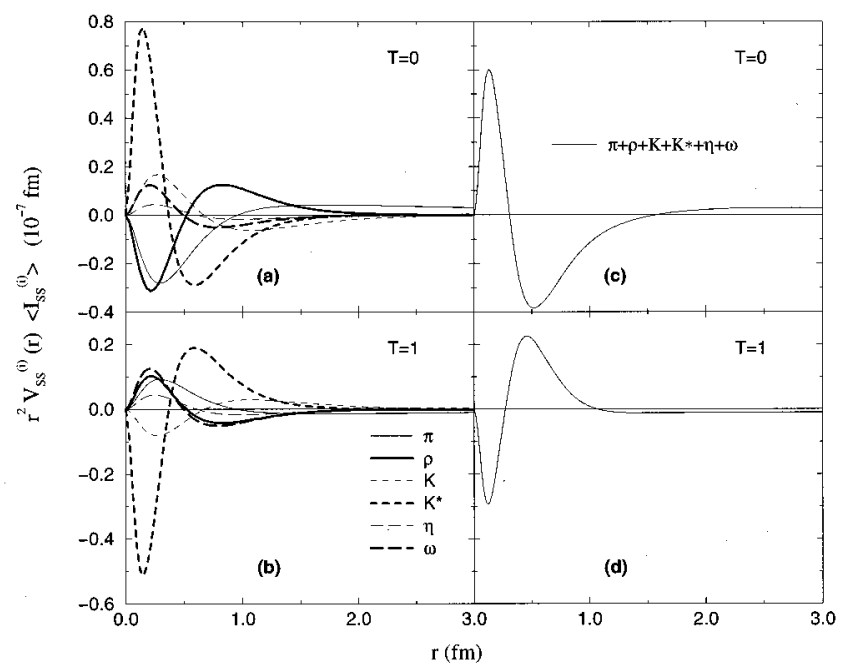

FIG. 4. Individual meson contributions to the isoscalar (a) and isovector (b) central spin dependent regularized potential $r^{2} V_{S S}^{\mu}(r)$. The potential obtained by adding all meson contributions for the isoscalar (c) and isovector (d) cases.

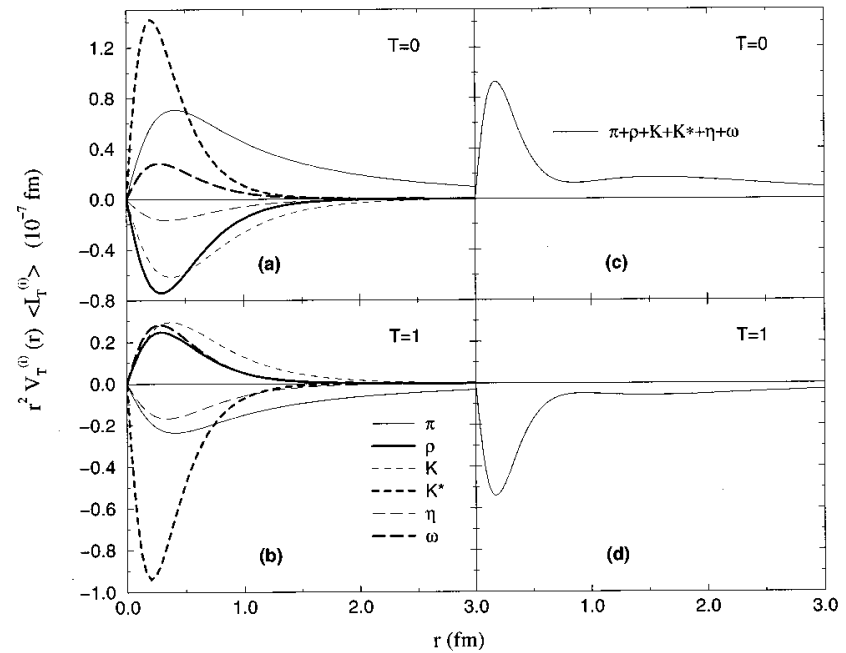

FIG. 5. Individual meson contributions to the isoscalar (a) and isovector (b) tensor regularized potential $r^{2} V_{T}^{\mu}(r)$. The potential obtained by adding all meson contributions for the isoscalar (c) and isovector (d) cases.

pair and destructive for the $(\pi, \rho)$ pair in both isospin channels. The $\left(K, K^{*}\right)$ pair shows a destructive interference in the $T=0$ channel and a constructive one in the $T=1$ channel. In these figures the longest range of the pion contribution stands out quite clearly over the other mesons, especially in the $T=0$ channel.

\section{RESULTS}

We used a hypertriton wave function based on the Nijmegen $93 N N$ potential [13] and the Nijmegen $Y N$ interaction [2], which include the $\Lambda-\Sigma$ transitions. The number of channels (see Sec. III) used in the solution of the corresponding Faddeev equation is 102 . This leads to a fully converged state, which has the proper antisymmetrization among the two nucleons built in. Also the $N N$ and $Y N$ cor-

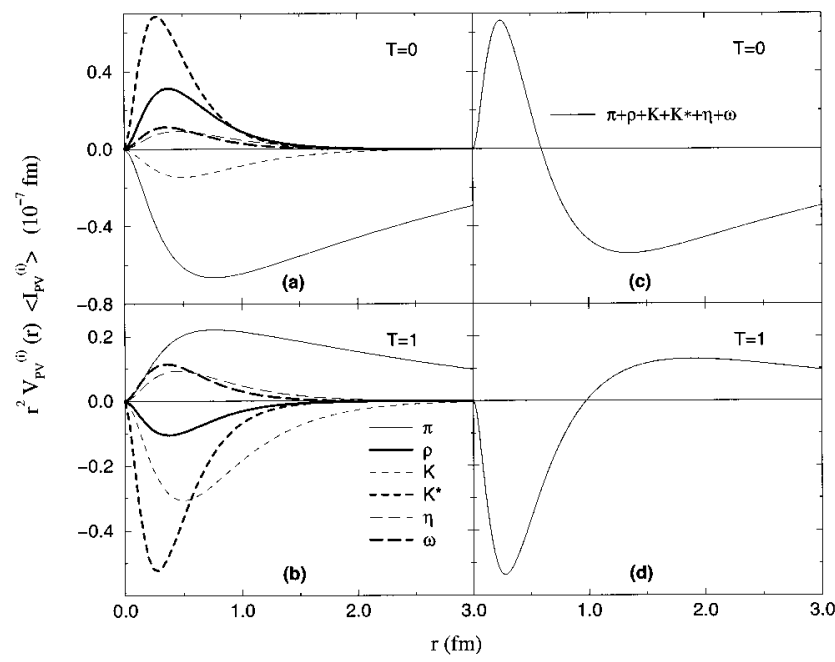

FIG. 6. Individual meson contributions to the isoscalar (a) and isovector (b) parity-violating regularized potential $r^{2} V_{\mathrm{PV}}^{\mu}(r)$. The potential obtained by adding all meson contributions for the isoscalar (c) and isovector (d) cases. 
TABLE III. Decay rates in units of $s^{-1}$ for individual meson exchanges and for partially summed up exchanges.

\begin{tabular}{llcl}
\hline \hline & $\Gamma^{\text {meson }}$ & \multicolumn{2}{c}{$\Gamma^{\text {partially summed }}$} \\
\hline$\pi$ & $0.2412 \times 10^{8}$ & $\pi$ & $0.2412 \times 10^{8}$ \\
$\eta$ & $0.4826 \times 10^{6}$ & $\pi+\eta$ & $0.2299 \times 10^{8}$ \\
$K$ & $0.5422 \times 10^{7}$ & $\pi+\eta+K$ & $0.9267 \times 10^{7}$ \\
$\rho$ & $0.7647 \times 10^{6}$ & $\pi+\eta+K+\rho$ & $0.7502 \times 10^{8}$ \\
$\omega$ & $0.4372 \times 10^{7}$ & $\pi+\eta+K+\rho+\omega$ & $0.1752 \times 10^{8}$ \\
$K^{*}$ & $0.5569 \times 10^{7}$ & $\pi+\eta+K+\rho+\omega+K^{*}$ & $0.2126 \times 10^{8}$ \\
\hline \hline
\end{tabular}

relations are exactly included as generated by the various baryon-baryon forces (see [1]). The $\Sigma N N$ part of the state has a probability of $0.5 \%$ and will be neglected.

The deuteron and the final state interaction among the three nucleons is generated using the Nijmegen $93 \mathrm{NN}$ force, including the $N N$ force components up to total two-body angular momentum $j=2$. This is sufficient to get a converged result for the nuclear matrix element.

Since the total three-body angular momentum is conserved, the Faddeev equation (20) for the final state interaction (FSI) has to be solved only for total three-body angular momentum $J=\frac{1}{2}$, but for both parities due to the parityviolating transition potential.

The total nonmesonic decay rate turns out to be $\Gamma=0.21 \times 10^{8}(1 / s)$, which is $0.55 \%$ of the free $\Lambda$ decay rate, $\Gamma_{\Lambda}=3.8 \times 10^{9}(1 / s)$. This is about 1 order of magnitude smaller than what has been found in the rough estimate [4], which was based only on $\pi$ exchange, a simplified hypertriton wave function, and the absence of FSI. In the following, we show that the final value for the total decay rate comes from many dynamical ingredients, which all contribute significantly. Therefore, that quantity will be an important test for our understanding of that system and should be measured.

Table III shows the individual contributions of the six mesons to $\Gamma_{n m}$ and the way each meson contributes to the final result. We see that the pion by itself provides the largest contribution, followed by $K, K^{*}$, and $\omega$. Adding the meson contributions one by one can yield a strongly varying sequence as seen in Table III choosing a special but arbitrary order. In view of Figs. 3-6 this is hardly surprising. The final result, however, is such that one ends up close to the value with pion exchange only.

The total decay rate is the sum of the partial rates for the

TABLE IV. Selected decay rates in units of $s^{-1}$ for $\pi$ exchange only and for exchange of all mesons.

\begin{tabular}{lcc}
\hline \hline & $\pi$ exchange only & Exchange of all mesons \\
\hline$\Gamma_{\mathrm{PWIAS}}^{n+d}$ & $0.59 \times 10^{7}$ & $0.47 \times 10^{7}$ \\
$\Gamma^{n+d}$ & $0.15 \times 10^{7}$ & $0.22 \times 10^{7}$ \\
$\Gamma_{\mathrm{PWIAS}}^{n+n+p}$ & $0.46 \times 10^{8}$ & $0.36 \times 10^{8}$ \\
$\Gamma^{n+n+p}$ & $0.23 \times 10^{8}$ & $0.19 \times 10^{8}$ \\
$\Gamma_{\mathrm{PC}}^{n+d}$ & $0.88 \times 10^{6}$ & $0.22 \times 10^{7}$ \\
$\Gamma_{\mathrm{PV}}^{n+d}$ & $0.59 \times 10^{6}$ & $0.22 \times 10^{5}$ \\
$\Gamma_{\mathrm{PC}}^{n+p+p}$ & $0.13 \times 10^{8}$ & $0.12 \times 10^{8}$ \\
$\Gamma_{\mathrm{PV}}^{n+p+p}$ & $0.92 \times 10^{7}$ & $0.73 \times 10^{7}$ \\
\hline \hline
\end{tabular}

TABLE V. Proton- and neutron-induced decay rates in units of $s^{-1}$ for $\pi$ exchange only and for the exchange of all mesons in comparison to the total $n d$ and $n n p$ rates.

\begin{tabular}{lcc}
\hline \hline & $\pi$ exchange only & Exchange of all mesons \\
\hline$\Gamma_{(n)}^{n+d}$ & $0.20 \times 10^{6}$ & $0.64 \times 10^{6}$ \\
$\Gamma_{(p)}^{n+d}$ & $0.78 \times 10^{6}$ & $0.70 \times 10^{6}$ \\
$\Gamma^{n+d}$ & $0.15 \times 10^{7}$ & $0.22 \times 10^{7}$ \\
$\Gamma_{(n)}^{n+n+p}$ & $0.59 \times 10^{7}$ & $0.57 \times 10^{7}$ \\
$\Gamma_{(p)}^{n+n+p}$ & $0.18 \times 10^{8}$ & $0.13 \times 10^{8}$ \\
$\Gamma^{n+n+p}$ & $0.23 \times 10^{8}$ & $0.19 \times 10^{8}$ \\
\hline \hline
\end{tabular}

$n d$ and $n n p$ decays. Our result for the pion only is shown in Table IV. The $n n p$ contribution is clearly dominant. We also show the plane wave impulse approximation (symmetrized) (PWIAS) results. They are defined by evaluating the nuclear matrix elements in Eqs. (19) and (21) keeping only the first terms, respectively. The comparison of PWIAS to the full result [keeping both terms in Eqs. (19) and (21)] underlines the importance of the final state interaction, which reduces the rate. Finally the parity-conserving and parity-violating contributions are listed and it is seen that they are comparable to each other, though with a slight dominance of the parity-conserving part.

The corresponding numbers including all mesons are also displayed in Table IV. Again the final state interaction is very important and reduces the PWIAS results by about a factor of 2. Now for all mesons included the parityconserving part is clearly dominant.

There is often a separation of $p$ - and $n$-induced decay in the literature [5,7]. They act clearly coherently and strictly spoken cannot be separated experimentally. Theoretically, however, we can choose in the intermediate state in front of $\left|\Psi_{{ }^{3} \mathrm{H}}\right\rangle$ in Eqs. (19), (20), and (21), a situation that the $\Lambda$ particle chooses either a proton or a neutron as its partner for meson exchanges. This amounts to keeping only the first or second term on the right hand side of Eq. (30), respectively. As already mentioned above this requires one to keep also $T=3 / 2$ states in the final state.

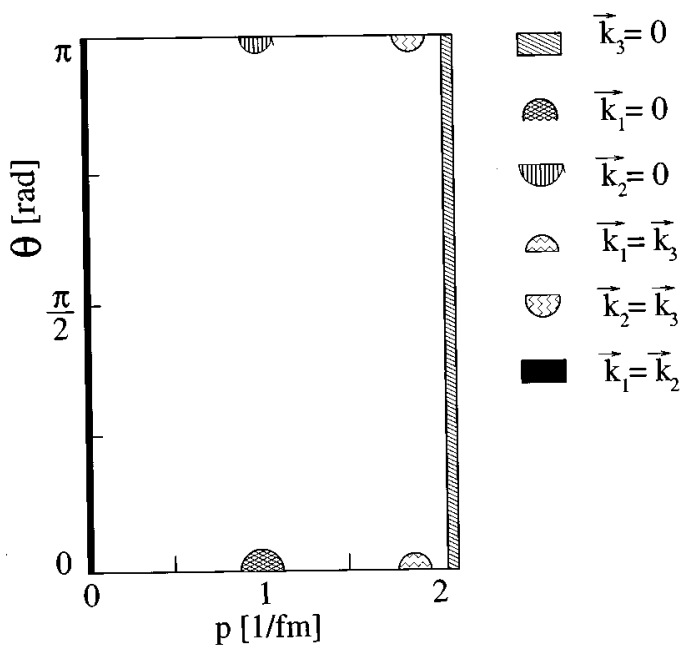

FIG. 7. The location of the three peaks corresponding to $\vec{k}_{i}=0(i=1,2,3)$ and the FSI peaks in the $\theta-p$ plane (see text). 
Our results for the pion only and for all six mesons are displayed in Table V. For the $n d$ breakup clearly the separate $n$ - and $p$-induced decay rates do not add up to the total $n d$-decay rate, which tells that there is interference. On the other hand, for the $n n p$ breakup the separate contributions from the $n$ - and $p$-induced decays add up to the total $n n p$-decay rate. However, this does not imply automatically that they can be separated experimentally. We come back to that interesting issue below. We see that for $n d$ and $n n p$ decay the $p$-induced decay is stronger.

Focusing on the PWIAS for the $n n p$ breakup of Eq. (19), one has three contributions

$$
\begin{aligned}
\left\langle\phi_{0}^{a}|(1+P) \hat{O}(1,2)| \Psi_{\Lambda_{H}}\right\rangle= & \frac{1}{\sqrt{6}}{ }_{12}^{a}\left\langle\left.\vec{p} m_{1} m_{2} \nu_{1} \nu_{2}\right|_{3}\left\langle\vec{q} m_{3} \nu_{3}|\hat{O}(1,2)| \Psi_{{ }^{3}} \mathrm{H}\right\rangle+\frac{1}{\sqrt{6}} \stackrel{a}{23}\left\langle\left.\vec{p} m_{1} m_{2} \nu_{1} \nu_{2}\right|_{1}\left\langle\vec{q} m_{3} \nu_{3}|\hat{O}(1,2)| \Psi_{\Lambda^{H}}\right\rangle\right.\right. \\
& +\frac{1}{\sqrt{6}} \stackrel{a}{31}\left\langle\left.\vec{p} m_{1} m_{2} \nu_{1} \nu_{2}\right|_{2}\left\langle\vec{q} m_{3} \nu_{3}|\hat{O}(1,2)| \Psi_{{ }^{3} \mathrm{H}}\right\rangle .\right.
\end{aligned}
$$

In the above equation we applied the $P$ operator to the left. As always, the subscripts refer to the particles in states with momenta $\vec{p}, \vec{q}$, spin magnetic quantum numbers $m$, and neutron or proton labels $\nu$. Using Eq. (30) the isospin matrix elements can simply be calculated with the result that in the first matrix element on the right-hand side of Eq. (55) nucleons 1 and 2 are two neutrons for $n$-induced decay and a neutron-proton pair for the $p$-induced decay. Also that first matrix element peaks at $\vec{q}=0$, which means that nucleons 1 and 2 share the total energy and emerge back to back. Under this kinematical condition the other two matrix elements are strongly suppressed, as is manifest if one expresses the momenta occurring in these two matrix elements in terms of $\vec{p}$ and $\vec{q}=0$ of the first matrix element. If we denote the $\vec{p}$ from the first matrix element as $\vec{p}_{12}$, then it turns out that $\vec{p}=-\frac{1}{2} \vec{p}_{12}\left(-\frac{1}{2} \vec{p}_{12}\right)$ and $\vec{q}=\vec{p}_{12}\left(-\vec{p}_{12}\right)$ in the second (third) matrix element, respectively, and for such a $\vec{q}$ value $\left|\Psi_{{ }^{3} \mathrm{H}}\right\rangle$ is suppressed.

The other two matrix elements also peak if particles 1 or 2 emerge with zero momenta. Therefore, we have to expect three peaks. Let us now take a closer look at the quantity $d \Gamma^{n+n+p} / d p d \theta$, defined in Eq. (10). That quantity, suitably restricted to certain or all meson exchanges, summed over all $p$ and $\theta$ values provided the various values of Tables III-V. For the choice of Jacobi momenta (4) the three peaks are located as sketched in Fig. 7. Energy and momentum conservation requires that $p^{2}+\frac{3}{4} q^{2} \equiv p_{\max }^{2}$, where $p_{\max }$ is the maxi-

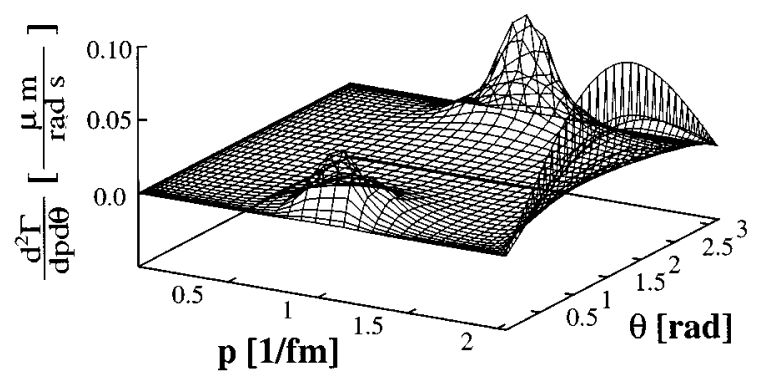

FIG. 8. The differential decay rate $d \Gamma^{n+n+p} / d p d \theta$ for PWIAS and exchange of all mesons. mal $p$ value. For the available energy $p_{\max } \approx 2 \mathrm{fm}^{-1}$. As an example we regard $\vec{k}_{2}=0$. Then $\vec{p}=\frac{1}{2} \vec{k}_{1}$ and $\vec{q}=-\vec{k}_{1}$. It follows that $k_{1}=p_{\max }$ and consequently $p=\frac{1}{2} p_{\max }$. For $\vec{k}_{3}=0$ the momenta $\vec{k}_{1}$ and $\vec{k}_{2}$ have to be back to back and there will be no $\theta$ dependence; for $\vec{k}_{2}=0$ the momenta $\vec{k}_{3}$ and $\vec{k}_{1}$ are opposite to each other, therefore $\theta=\pi$ and finally for $\vec{k}_{1}=0$ the momenta $\vec{k}_{3}=-\vec{k}_{2}$ and $\theta=0$. For PWIAS the result is displayed in Fig. 8 for the exchange of all mesons. The corresponding results for $\pi$ exchange only is qualitatively similar but larger by about $50 \%$. As expected we see the three peaks at the proper locations. The variation with $\theta$ for the maximal $p$ value is due to the factor $\sin \theta$ in expression (10). Note that the $\sin \theta$ dependence also removes the highest peak values for all three peaks.

For the choice of nucleons 1 and 3 being neutrons and 2 a proton we thus have to expect that for a neutron-induced decay there should be only one peak at the position $p$ $=\frac{1}{2} p_{\max }$ and $\theta=\pi$, which is indeed the case as shown in Fig. 9. Note that in evaluating the nuclear matrix element of Eq. (10) we fixed the isospin magnetic quantum numbers $\nu_{1}$, $\nu_{2}, \nu_{3}$ to be $-\frac{1}{2}, \frac{1}{2},-\frac{1}{2}$. This refers to Figs. 8-13. For $p$-induced decay we expect two peaks corresponding to either $\vec{k}_{1}=0$ or $\vec{k}_{2}=0$. And this is what comes out and what is shown in Fig. 10. Regarding Figs. 9 and 10 we see that the areas populated by $n$ - and $p$-induced decays appear to be well separated in phase space and seem to add up essentially

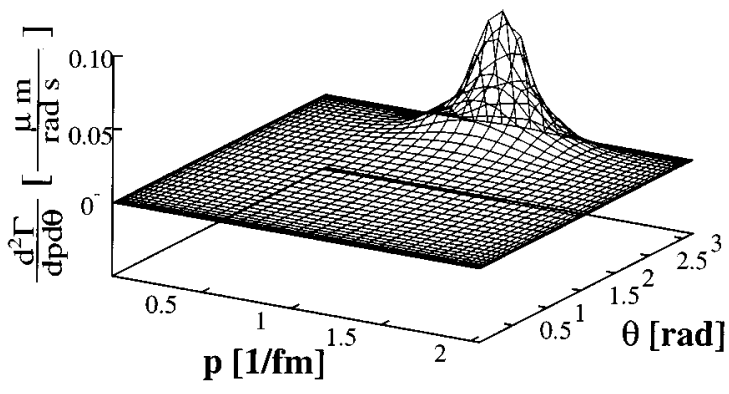

FIG. 9. The $n$-induced differential decay rate $d \Gamma_{(n)}^{n+n+p} / d p d \theta$ for PWIAS and $\pi$ exchange only. Nucleons 1, 2, and 3 are chosen to be a neutron, a proton, and a neutron. 


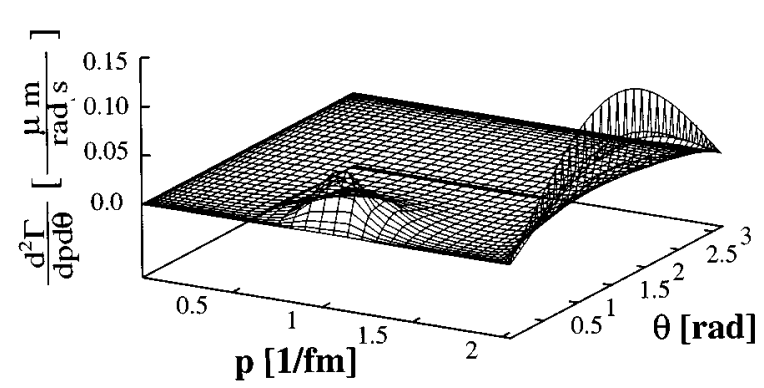

FIG. 10. The same as in Fig. 9 for $p$-induced decay.

incoherently to the full result. A closer inspection, however, will be carried through below, which leads to a different result.

Now let us turn on the final state interaction. For three nucleons interacting among each other one knows from $\mathrm{Nd}$ breakup reactions [9] that cross sections are strongly enhanced if two nucleons emerge with equal momenta. This is due to the strong interaction in the ${ }^{1} S_{0}$ state, where the $N N t$ matrix has a pole close to zero energy (virtual state). These enhancements will be called final state interaction peaks (FSIP's) in the following. Figure 7 shows the positions where this happens in the $p-\theta$ plane. For the case $p=0$ clearly no $\theta$ dependence is present. For all meson exchanges Fig. 11 shows $d \Gamma^{n+n+p} / d p d \theta$ including the full final state interaction. We see again the three peaks already known from the PWIAS result, but with reduced heights according to the already known reduction of the rate due to the final state interaction. In addition there are two more little peaks caused by the final state interaction for two pairs of nucleons, where the nucleons forming a pair have equal momenta, respectively. The final state interaction peak for the third pair is suppressed by the kinematical factor $p^{2}$ in Eq. (10). The $p$ - and $n$-induced pictures (Figs. 12 and 13) keeping the full final state interaction again look qualitatively similar to the ones evaluated in the PWIAS approximation, only the final state interaction peaks are added.

It is interesting to see despite the fact that FSI decreases the $d \Gamma^{n+n+p} / d p d \theta$ values significantly, which means a strong rescattering among the three nucleons, that there is only one peak for the $n$-induced decay and the rescattering does not populate the other two peak areas. The corresponding is also true for the $p$-induced decay. Again the events for $n$ - and $p$-induced decays seem to add up incoherently in the quantity $d \Gamma^{n+n+p} / d p d \theta$.

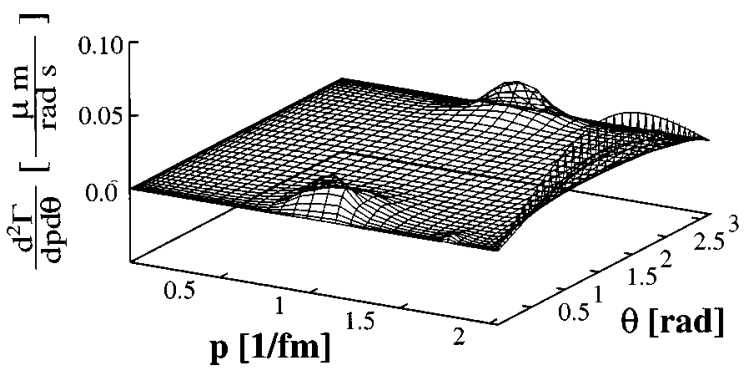

FIG. 11. The differential decay rate $d \Gamma^{n+n+p} / d p d \theta$ with full inclusion of the final state interaction and exchange of all mesons.

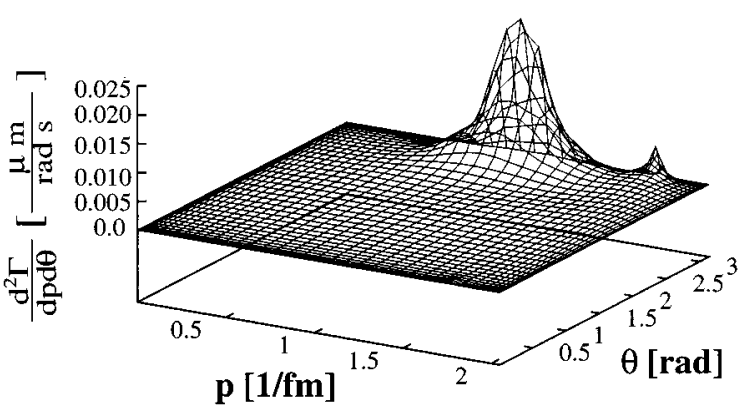

FIG. 12. The $n$-induced differential decay rate $d \Gamma_{(n)}^{n+n+p} / d p d \theta$ with full inclusion of the final state interaction and $\pi$-exchange only.

Let us now discuss the form (11) of the differential decay rate expressed in individual momenta in the total momentum zero frame of the decaying hypertriton. After averaging over the initial state polarization and summing over the spin magnetic quantum numbers of the final three nucleons, the decay rate can depend only on the angle between the two nucleon detectors, $\Theta_{12}$. We now show two sets of figures, Figs. 14 and 15 , for various $\Theta_{12}$ 's $0^{\circ} \leqslant \Theta_{12} \leqslant 180^{\circ}$ and for pion exchange only. In Fig. 14 we compare PWIAS to the full calculation including final state interaction. Thereby the two detected nucleons can be either a proton-neutron pair or two neutrons. For $\Theta_{12}=180^{\circ}$ we see a strong enhancement for $S \approx 110 \mathrm{MeV}$. (This corresponds to a location around the middle of the locus in Fig. 1.) In PWIAS this is caused by the fact that there the ${ }_{\Lambda}^{3} \mathrm{H}$ wave function enters at $\vec{q}=\vec{k}_{3}=0$. As in Nd scattering, we shall from now on call such a configuration, where one final nucleon has zero momentum, a quasifree scattering (QFS) configuration. The final state interaction reduces that enhancement, but it is still pronounced. In addition we see two FSIP's in the full calculation. (They have to be absent, of course, in PWIAS.) For $\Theta_{12}=160^{\circ}$ that enhancement is reduced and two peaks emerge at the beginning and the end of the $S$ curve. Since there either $E_{1}$ or $E_{2}$ are small, we have again configurations, which are close to QFS conditions, now for the nucleon pairs 2,3 and 1,3 , respectively. This explains the additional enhancements. Now at $\Theta_{12}=120^{\circ}$ the enhancement in the middle of the $S$ curve has disappeared. That point on the $S$ curve corresponds exactly to the so-called space-star configuration in an $N+d \rightarrow N+N+N$ process. All three nucleons receive the same energy and emerge com-

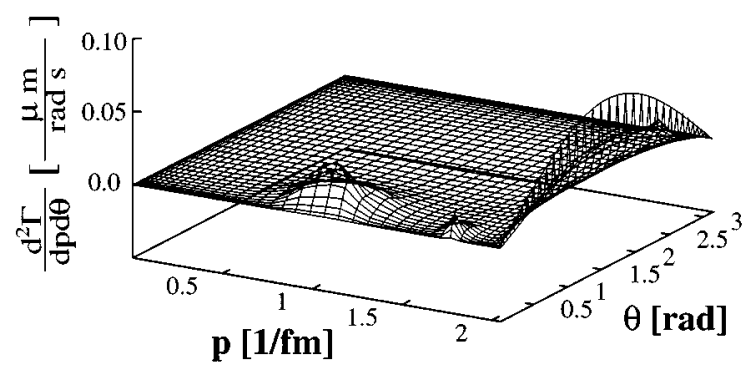

FIG. 13. The same as in Fig. 12 for $p$-induced decay. 

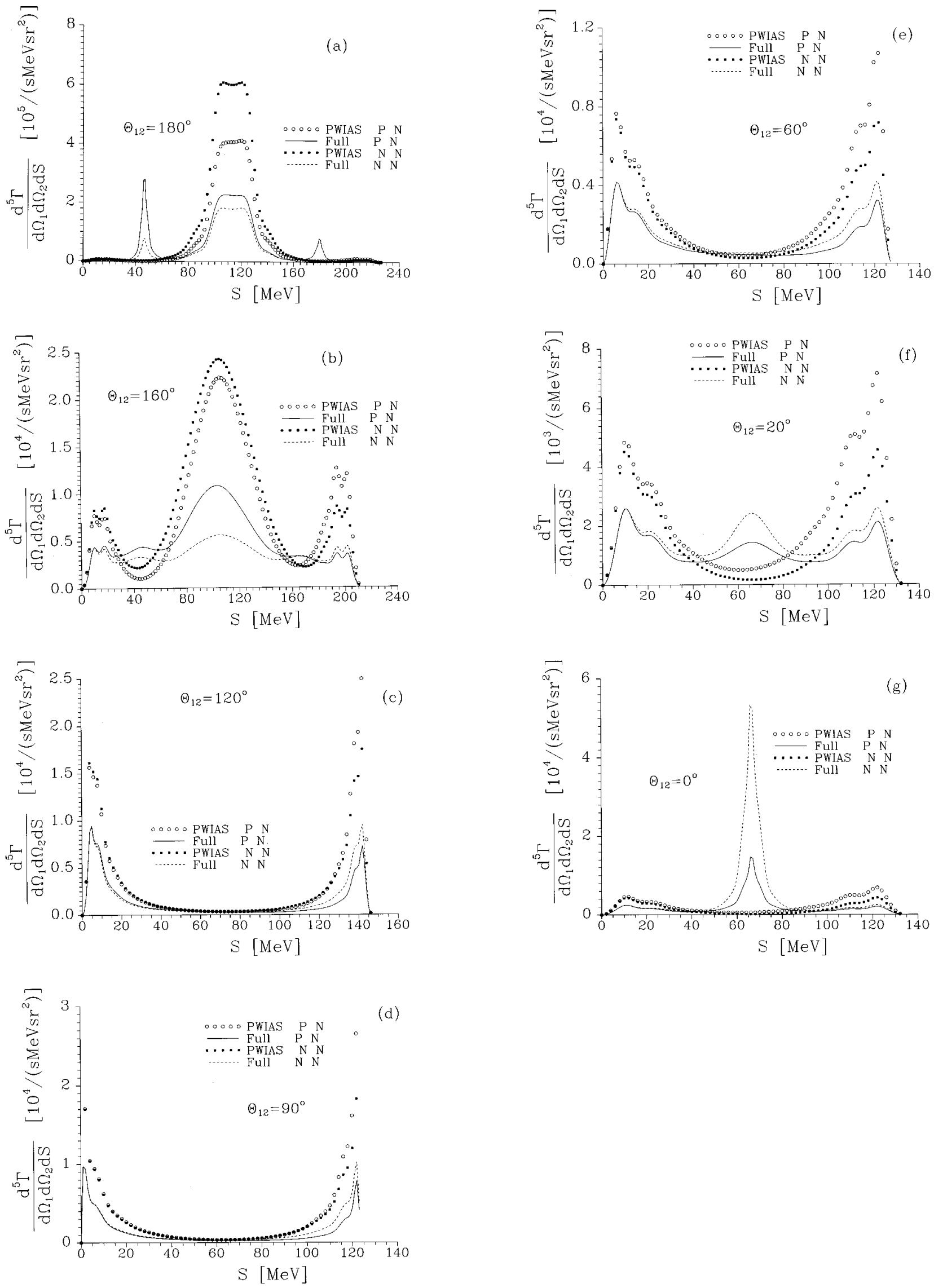

FIG. 14. The differential decay rate $d \Gamma^{n+n+p} / d \Theta_{12} d S$ for various angles $\Theta_{12}$ and $\pi$ exchange only. The two detected nucleons are either a $p$ (particle 1) $n$ (particle 2) pair or two neutrons. PWIAS is compared to the treatment including the full final state interaction. 

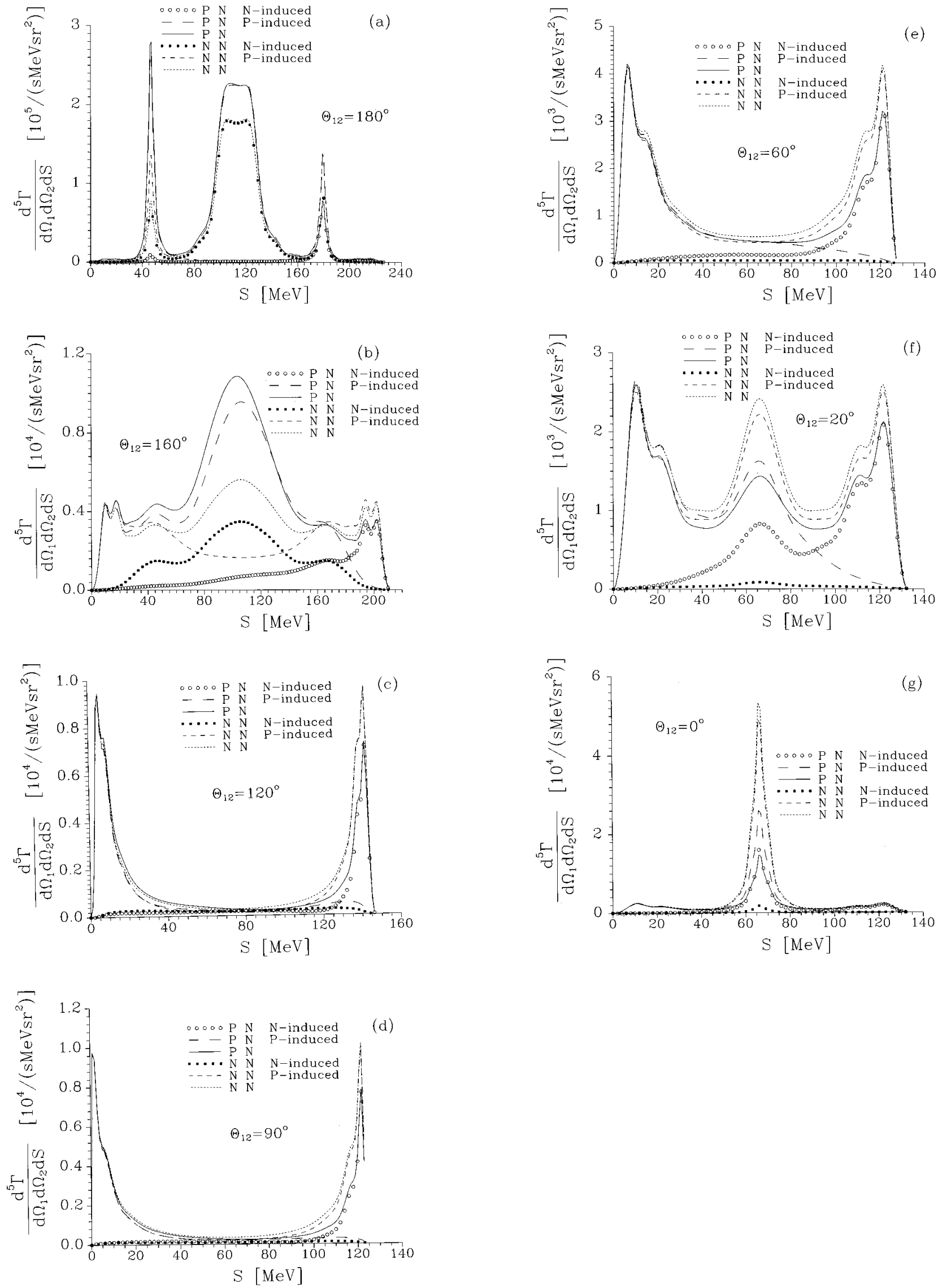

FIG. 15. The $n$ - and $p$-induced differential decay rates $d \Gamma_{(n),(p)}^{n+n+p} / d \Theta_{12} d S$ in comparison to the physical rate for various angles $\Theta_{12}$ and $\pi$ exchange only. The two detected nucleons are either a $p$ (particle 1) $n$ (particle 2) pair or two neutrons. The final state interaction is fully included. 

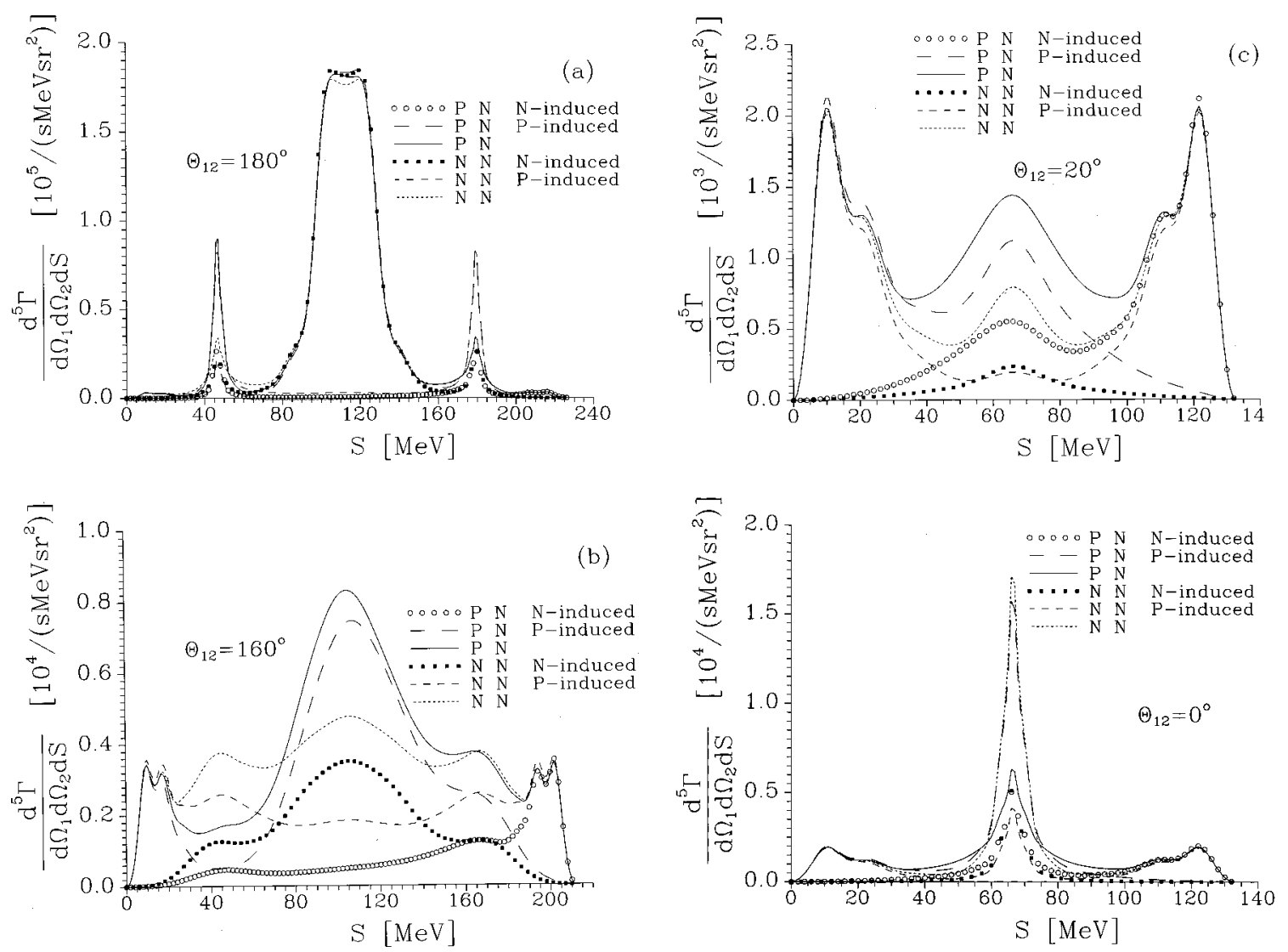

FIG. 16. The same as in Fig. 15 for the exchange of all mesons.

pletely symmetrically under $120^{\circ}$ pairwise angles. This is far away from QFS conditions and no enhancement is expected. Of course, at the beginning and end of the $S$ curve the peaks correspond again to conditions close to QFS. The situation remains similar at $\Theta_{12}=90^{\circ}$ and $60^{\circ}$. Finally a new structure appears at $\Theta_{12}=20^{\circ}$ and above all at $\Theta_{12}=0^{\circ}$ in the middle of the $S$ curve. This is a FSIP, which is fully developed for $\Theta_{12}=0^{\circ}$. In principle it could be used to extract information on the $n p$ and $n n$ scattering lengths like in Nd breakup processes.

In Fig. 15 we compare the full calculations to the separate decay rates for $n$ - and $p$-induced processes. Note that in this figure PN means that the proton is nucleon 1 and a neutron is nucleon 2, which corresponds to $\nu_{1}=\frac{1}{2}, \quad \nu_{2}=-\frac{1}{2}$, and $\nu_{3}=-\frac{1}{2}$ in the matrix element of Eq. (11).

At $\Theta_{12}=180^{\circ}$ the decay rates in the center of the $S$ curve are essentially given by the $p$-induced process if a proton neutron pair is registered and by the $n$-induced process if two neutrons are registered. Already at $\Theta_{12}=160^{\circ}$ this is no longer true. The $p$-induced rate and even more the $n$-induced rate is significantly lower than the rate built up by the full physical process. It is interesting to see in cases of Figs. 15(b)-15(f) that the $n$-induced decay rate for $p n$ detection at the upper end of $S$ is practically identical to the decay rate corresponding to the full physical process. The reason is that at the upper end of $S$ the energy $E_{1}$ (the proton energy) is nearly zero (see Fig. 1), and therefore two neutrons carry essentially all the energy. Without FSI's such a case can only be generated by an $n$-induced decay and qualitatively this picture does not change due to FSI's. In the case of $p$-induced decay, its rate for $p n$ and $n n$ detection at the lower end of $S$ is practically identical with the full decay rate. The reason is similar as for the $n$-induced decay. At the lower end of $S$ the energy $E_{2}$ (a neutron energy) is nearly zero. Therefore a proton-neutron pair has to carry essentially all the energy and this has to be generated by a $p$-induced process. All these enhancements at the lower and upper end of $S$ are QFS-like cases.

Interesting are also the FSIP's, especially pronounced at $\Theta_{12}=0^{\circ}$ and $180^{\circ}$. At $\Theta_{12}=0^{\circ}$ and for neutron-neutron detections the proton has to fly in the opposite direction, therefore the $p$-induced process has to be mainly responsible for the peak, as is the case. For $p-n$ detection, however, both $p$ - and $n$-induced decays can contribute to a FSIP and they do. Apparently the $p$ - and $n$-induced decays have to interfere, since the individual rates do not add up to the total physical decay. As a further example we comment on the left strong FSIP at $\Theta_{12}=180^{\circ}$. There are two peaks according to $p n$ and $n n$ detections. For $n n$ detection (the neutrons have opposite momenta) this has to be necessarily a $p n$ FSIP. As we see from the figure it receives contributions from $n$ - and $p$-induced processes, again coherently. In the case of $p n$ detection (the proton an a neutron have opposite momenta) the special location on the $S$ curve (high proton energy) requires that it is an $n n$ FSIP. Therefore the very dominant contribution has to come from the $p$-induced process. This is clearly visible in Fig. 15.

The corresponding curves, when all mesons are included, are qualitatively the same and only very few examples are displayed in Fig. 16. The heights of the FSIP's have 
changed, however, significantly.

Finally we consider the question whether the total $n$ - and $p$-induced decay rates can be separated experimentally. As we already saw this is not possible in the $n d$ decay channel. There the two processes interfere coherently and the individual theoretical rates do not sum up to the total rate. In the $3 N$ decay channel the total rate is very close to the sum of the individual rates for the $n$ - and $p$-induced processes. Also, in the $p-\theta$ representation of $d \Gamma$ [see Eq. (10)] and displayed in Figs. 12 and 13, the events from the two different processes appear to be nicely separated. On the other hand, in the $\Theta_{12}-S$ representation, which is directly accessible using two detectors, we saw cases where an interference was manifest. The total decay rate into three nucleons is

$$
\begin{aligned}
\Gamma^{n+n+p} & =\int \frac{d^{5} \sigma}{d \hat{k}_{1} d \hat{k}_{2} d S} d \hat{k}_{1} d \hat{k}_{2} d S \\
& =8 \pi^{2} \int_{0}^{\pi} d \Theta_{12} \sin \Theta_{12} \int_{0}^{S_{\max }\left(\Theta_{12}\right)} d S \sigma\left(\Theta_{12}, S\right) .
\end{aligned}
$$

We used the fact that $d^{5} \sigma / d \hat{k}_{1} d \hat{k}_{2} d S$ depends only on $\Theta_{12}$ and $S$ and introduced the length $S_{\max }\left(\Theta_{12}\right)$ of the $S$ curve depending on $\Theta_{12}$. First of all we notice immediately that the pure QFS cases, where a final nucleon momentum is zero, do not contribute, since for $\vec{k}_{3}=0 \Theta_{12}=180^{\circ}$ and for $\vec{k}_{1}=0$ or $\vec{k}_{2}=0$ the phase space factor in Eq. (11) is zero. Nevertheless an angular configuration with $\Theta_{12}=180^{\circ}$ can and should be measured by itself, since along the $S$ curve there will be one point with the exact QFS conditions and as we saw in Fig. 15 there the $n$ - and $p$-induced processes can be cleanly separated. One measures either a $p n$ or an $n n$ pair and they are generated by $p$ - and $n$-induced decays, respectively.

We now discuss the quantity

$$
\gamma\left(\Theta_{12}\right)=8 \pi^{2} \sin \Theta_{12} \int_{0}^{S_{\max }\left(\Theta_{12}\right)} d S \sigma\left(\Theta_{12}, S\right)
$$

for $n p$ and $n n$ detection, respectively. This is shown in Figs. 17 and 18 together with the individual contributions of the $p$ - and $n$-induced processes. We see strong peaks near $\Theta_{12}=170^{\circ}$ for the full processes. The corresponding values for the $p(n)$-induced decay are similar in the peak area for $p n(n n)$ detection, while the $n(p)$-induced values are small.

At smaller angles $\Theta_{12}$ the $p$ - and $n$-induced values are similar to each other in the case of $p n$ detections, while for $n n$ detection the $p$-induced quantities dominate. A closer inspection reveals that the sum of the $p$ - and $n$-induced values for each $\Theta_{12}$ do not sum up very well to the value according to the true physical process, but there are differences up to $10 \%$ ( $n n$ detection) around $\Theta_{12}=170^{\circ}$. This is a clear signal for interference.

Let us quantify this question. The representation (10) of $d \Gamma^{n+n+p} / d p d \theta$, which has been displayed in Figs. 8-13, yields the decay rates for the individual $n$ - and $p$-induced processes as well as for the full physical process (neutron and proton induced) when integrated over the whole $p-\theta$

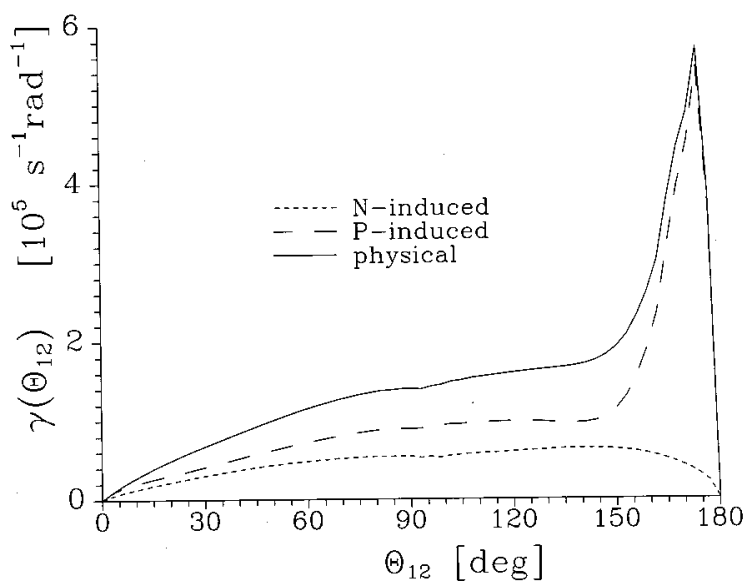

FIG. 17. Differential decay rates integrated over the $S$ curve as a function of $\Theta_{12}$. The $p$ - and $n$-induced cases are compared to the physical process. The final state interaction is fully included and all mesons are exchanged. The curves belong to the case that a proton and a neutron are detected.

plane. According to Figs. 12 and 13 the $n$ - and $p$-induced processes appear to receive their contributions from wellseparated areas in the $p-\theta$ plane. Quantitatively, however, this is not true. Restricting the integration in $\theta$ and $p$ to the region where the peaks in Figs. 12 and 13 are located results in only a certain fraction of the full rates. Quantitatively, if we fix that fraction to $60 \%$, say, for $p$ - and $n$-induced decays, respectively, we find that the regions displayed in Fig. 19 contribute. In choosing a certain fraction we always start integration from the highest values (located in the peaks) downwards and stop when the assumed fraction has been reached. Except for a small domain ( $\theta \approx \pi$ and $p$ large) the regions for $p$ - and $n$-induced decays are clearly separated. Of course for fractions smaller than $60 \%$ this will be even more the case. For fractions larger than $60 \%$, however, the regions overlap considerably. An example for $90 \%$ is also shown in Fig. 19. Clearly in such a case the events coming from for $p$ - and $n$-induced decays cannot be separated any more experimentally.

We show the fractional decay rates evaluated over corresponding increasing regions in Tables VI, VII, and in Figs.

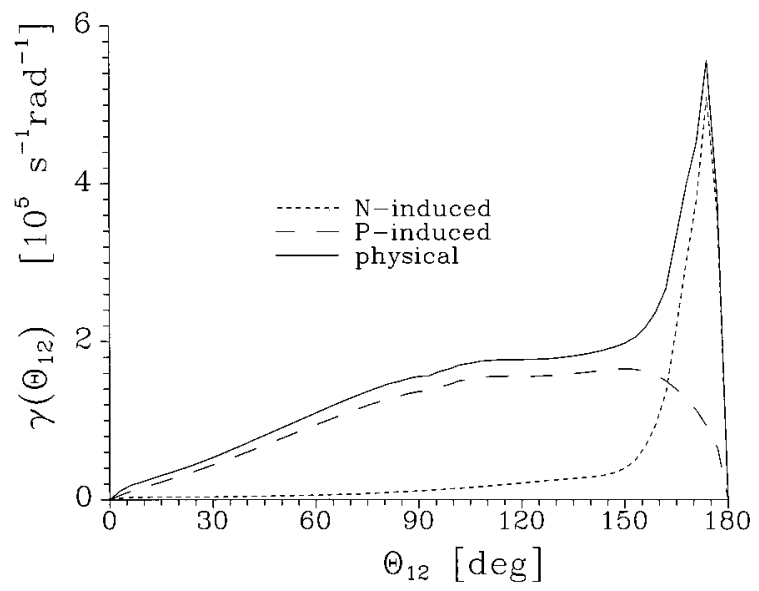

FIG. 18. The same as in Fig. 17 for the case that two neutrons are detected. 

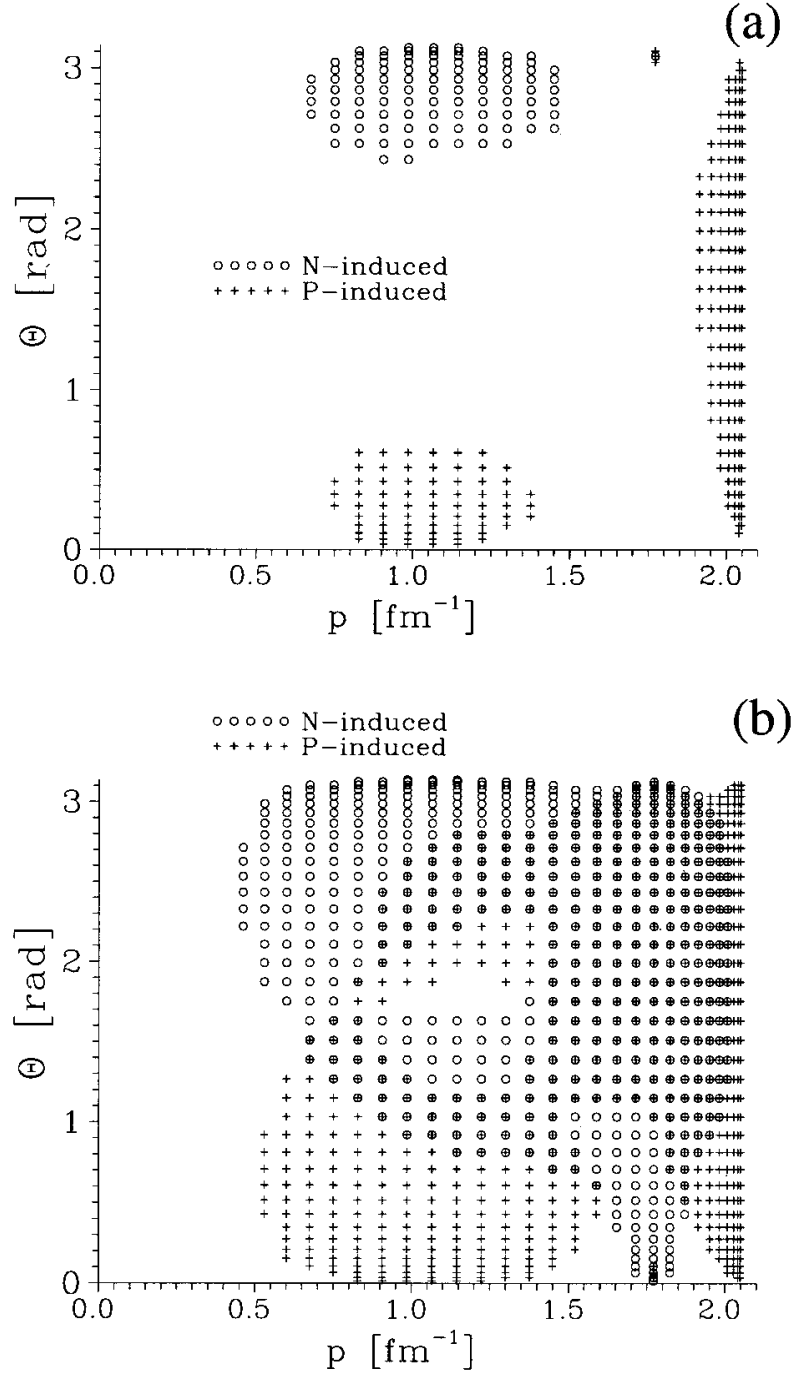

FIG. 19. The separate regions in the $\theta-p$ plane contributing to (a) $60 \%$ and (b) $90 \%$ of the rates of $n$ - and $p$-induced decays. Note the strong overlap of the different processes in phase space in case (b).

19 and 20, where the results refer to full calculations and include all meson exchanges. There the $p$ - and $n$-induced rates are compared to the observed rate $\Gamma_{\text {physical }}$, produced by the full physical process. Thus if we require that the measured value is equal to the $p$ - or $n$-induced decay within a few percent, one has to restrict the integration in the $\theta-p$ plane to certain subregions and the rates to only about $60 \%$ of the full rate.

TABLE VI. Fractional proton- and neutron-induced decay rates in units of $s^{-1} \Gamma_{(p),(n)}=\int d \Gamma_{(p),(n)}^{n+n+p}$ integrated over subdomains in the $\theta-p$ plane, where proton-induced decay dominates. They are compared to the corresponding fractional physical rate.

\begin{tabular}{lcccc}
\hline \hline Area $(P)$ & $\Gamma_{(p)}$ & $\Gamma_{(n)}$ & $\Gamma_{\text {physical }}$ & $\Gamma_{(p)} / \Gamma_{\text {physical }}$ \\
\hline $60 \%$ & $0.80 \times 10^{7}$ & $0.20 \times 10^{6}$ & $0.82 \times 10^{7}$ & $98 \%$ \\
$70 \%$ & $0.94 \times 10^{7}$ & $0.48 \times 10^{6}$ & $0.98 \times 10^{7}$ & $96 \%$ \\
$80 \%$ & $0.11 \times 10^{8}$ & $0.99 \times 10^{6}$ & $0.12 \times 10^{7}$ & $92 \%$ \\
$90 \%$ & $0.12 \times 10^{8}$ & $0.23 \times 10^{7}$ & $0.14 \times 10^{8}$ & $86 \%$ \\
\hline \hline
\end{tabular}

TABLE VII. Fractional proton- and neutron-induced decay rates $\Gamma_{(p),(n)}=\int d \Gamma_{(p),(n)}^{n+n+p}$ in units of $s^{-1}$ integrated over subdomains in the $\theta-p$ plane, where neutron-induced decay dominates. They are compared to the corresponding fractional physical rate.

\begin{tabular}{lcccc}
\hline \hline Area $(N)$ & $\Gamma_{(p)}$ & $\Gamma_{(\mathrm{n})}$ & $\Gamma_{\text {physical }}$ & $\Gamma_{(n)} / \Gamma_{\text {physical }}$ \\
\hline $60 \%$ & $0.34 \times 10^{6}$ & $0.34 \times 10^{7}$ & $0.35 \times 10^{7}$ & $97 \%$ \\
$70 \%$ & $0.11 \times 10^{7}$ & $0.40 \times 10^{7}$ & $0.47 \times 10^{7}$ & $85 \%$ \\
$80 \%$ & $0.29 \times 10^{7}$ & $0.45 \times 10^{7}$ & $0.72 \times 10^{7}$ & $63 \%$ \\
$90 \%$ & $0.50 \times 10^{7}$ & $0.51 \times 10^{7}$ & $0.10 \times 10^{8}$ & $51 \%$ \\
\hline \hline
\end{tabular}

Let us map the $\theta-p$ values into the variables $\Theta_{12}-S$, which are directly accessible experimentally. This is shown in Fig. 20 for 60 and $90 \%$. That picture refers to the detection of a neutron (particle 1)-proton (particle 2) pair. Figure 20 tells that $n$-induced decay can be found under all $\Theta_{12}$ angles for small $S$ values. ( $E_{2}$ is then small.) The $p$-induced decay on the other hand is to be found for all the $\Theta_{12}$ angles around maximal $S$ values and in the region $160^{\circ} \leqslant \Theta_{12} \leqslant 180^{\circ}$ for medium-large $S$ values. (For small neutron energies.) A correspondingly modified figure could be shown if two neutrons
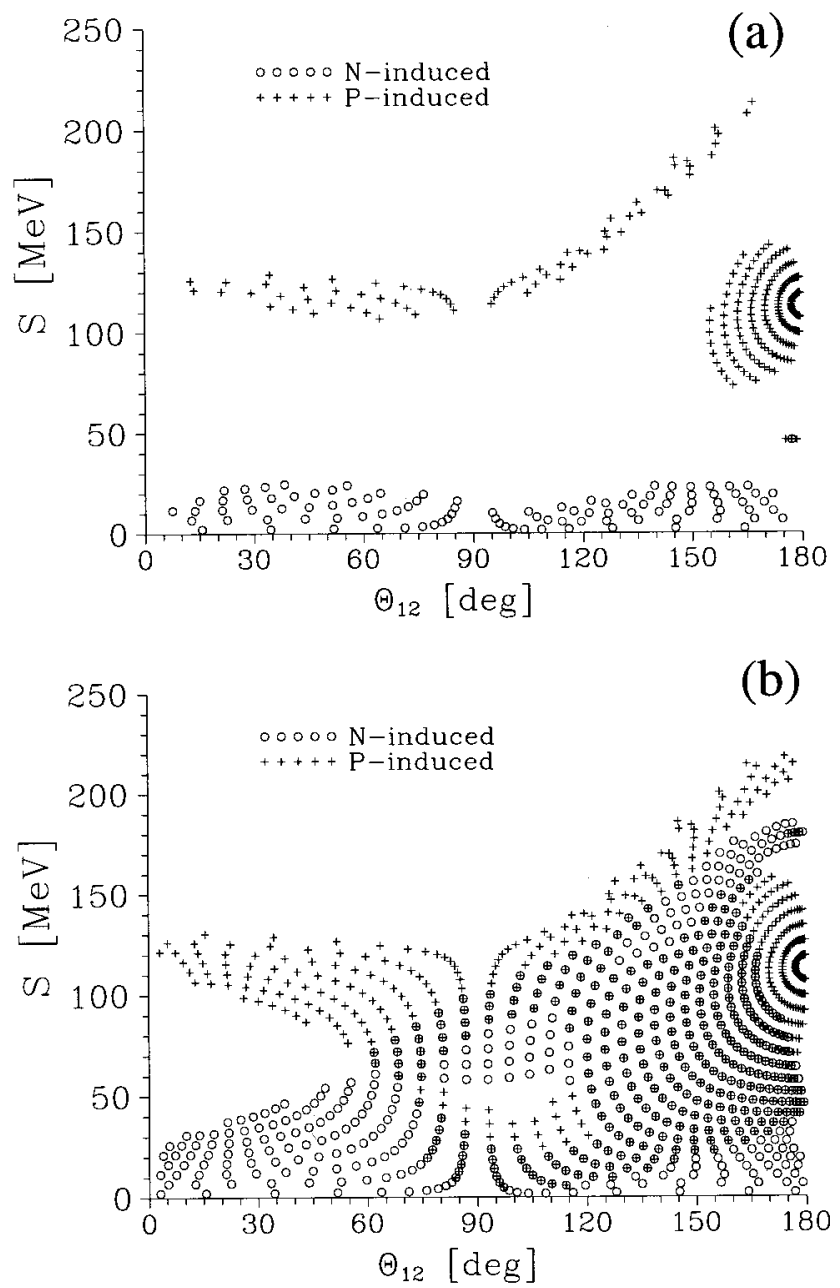

FIG. 20. The separate regions in the $\Theta_{12}-S$ plane contributing to (a) $60 \%$ and (b) $90 \%$ of the rates of $n$ - and $p$-induced decays. Note the strong overlap of the different processes in phase space in case (b). (Particle 1 is a neutron and particle 2 is a proton.) 
would be detected. If, on the other hand, $90 \%$ of the corresponding rates should be detected then the two detectors would receive events from both processes under the same angle $\Theta_{12}$ and for the same energies in a large portion of the phase space. Thus experimentally it is not possible to separate those processes.

We have to conclude that despite the fact that $\Gamma^{n+n+p}$ is rather close to the sum of $\Gamma_{(p)}^{n+n+p}$ and $\Gamma_{(n)}^{n+n+p}$, the latter values cannot be determined experimentally, only fractions, the smaller, the cleaner.

\section{SUMMARY AND CONCLUSIONS}

The nonmesonic hypertriton decay has been calculated based on rigorous solutions of three-body Faddeev equations for the hypertriton and the $3 N$ scattering states of the final three nucleons. Realistic $N N$ and hyperon-nucleon interactions have been used. In the meson exchange process the pion exchange is dominant, but the other included mesons $\eta, K, \omega, \rho$, and $\mathrm{K}^{*}$ also provide significant contributions of various signs and magnitudes. The final state interaction turned out to be very important and reduces the rates for PWIAS by about a factor of 2 . The total nonmesonic decay rate turns out to be $0.55 \%$ of the free $\Lambda$ decay rate and is smaller by an order of magnitude than a previous estimation [4] which used the pion-exchange model, a much more simplified hypertriton wave function and no FSI. While the $p$ and $n$-induced decays add up manifestly in a coherent manner in the $n d$ decay process, the $n n p$ decay rate is rather well given as the sum of the $n$ - and $p$-induced decay rates. Nevertheless these individual decay rates cannot be measured separately. Only fractions thereof can be obtained, when the contributions arise from nonoverlapping regions in phase space. This subject has been thoroughly discussed in Sec. V. Detailed information has been given regarding the separation of $p$ - and $n$-induced differential decay rates experimentally.

Since the decay rates depend sensitively on the number and type of mesons exchanged, it will be an interesting testground for the dynamics of these meson exchanges, which are driven by weak and a strong vertices. At the same time the decay rates probe the hypertriton wave function and the reaction mechanism of the three outgoing nucleons through their strong final state interaction. The latter one is especially manifest in the strong FSIP's, where two nucleons leave with equal momenta.

The evaluation of the pionic decay into the various bound states and continuum channels can be performed in a similar manner and is planned.

\section{ACKNOWLEDGMENTS}

This work was supported by the Polish Committee for Scientific Research under Grant No. PB 1031, the Science and Technology Cooperation Germany-Poland under Grant No. XO81.91, the DGICYT Grant No. PB92-0761 (Spain), the Generalitat de Catalunya Grant No. GRQ94-1022 and the US-DOE Grant No. DE-FG02-95-ER40907. A.P. acknowledges support from the Ministerio de Educación y Ciencia (Spain). The numerical calculations were performed on the Cray Y-MP of the Höchstleistungsrechenzentrum in Jülich, Germany.
[1] K. Miyagawa, H. Kamada, W. Glöckle, and V. G. J. Stoks, Phys. Rev. C 51, 2905 (1995).

[2] P. M. M. Maessen, Th. A. Rijken, and J. J. de Swart, Phys. Rev. C 40, 2226 (1989).

[3] G. Keyes et al., Nucl. Phys. B67, 269 (1973).

[4] C. Bennhold, A. Ramos, D. A. Aruliah, and U. Oelfke, Phys. Rev. C 45, 947 (1992).

[5] J. Cohen, Prog. Part. Nucl. Phys. 25, 139 (1990).

[6] J. F. Donoghue et al., Phys. Rep. 131, 319 (1986); L. de la Torre, Ph.D. thesis, Univ. of Massachusetts (1982); J. Dubach, G. B. Feldman, B. R. Holstein, and L. de la Torre, Ann. Phys. (N.Y.) 249, 146 (1996).

[7] A. Parreño, A. Ramos, and C. Bennhold (submitted to Phys. Rev. C).
[8] Particle Data Group, R. M. Barnett et al., Phys. Rev. D 54, 619 (1996), and references therein.

[9] W. Glöckle, H. Witala, D. Hüber, H. Kamada, and J. Golak, Phys. Rep. 274, 107 (1996).

[10] J. Golak, H. Kamada, H. Witala, W. Glöckle, and S. Ishikawa, Phys. Rev. C 51, 1638 (1995).

[11] W. Glöckle, The Quantum Mechanical Few-Body Problem (Springer, Berlin, 1983).

[12] R. Machleidt, K. Holinde, and Ch. Elster, Phys. Rep. 149, 1 (1987).

[13] V. G. J. Stoks, R. A. M. Klomp, C. P. F. Terhessen, and J. J. de Swart, Phys. Rev. C 49, 2950 (1994). 